\title{
Curcumin prevents and reverses murine cardiac hypertrophy
}

Hong-Liang Li, 1 Chen Liu, ${ }_{1}^{1}$ Geoffrey de Couto, ${ }^{1}$ Maral Ouzounian, ${ }^{1}$ Mei Sun ${ }^{1}$ Ai-Bing Wang, Yue Huang, ${ }^{3}$ Cheng-Wei He, ${ }^{4}$ Yu Shi, ${ }^{1}$ Xin Chen, ${ }^{1}$ Mai P. Nghiem, ${ }^{1}$ Youan Liu, ${ }^{1}$ Manyin Chen, ${ }^{1}$ Fayez Dawood, ${ }^{1}$ Masahiro Fukuoka, ${ }^{1}$ Yuichiro Maekawa, ${ }^{1}$ Liyong Zhang, ${ }^{1}$ Andrew Leask, ${ }^{5}$ Asish K. Ghosh, ${ }^{6}$ Lorrie A. Kirshenbaum, ${ }^{7}$ and Peter P. Liu'1,8

1Division of Cardiology, Heart and Stroke/Richard Lewar Centre of Excellence, University Health Network, University of Toronto, Toronto, Ontario, Canada. ${ }^{2}$ Laboratory of Molecular Cardiology, National Heart, Lung, and Blood Institute, NIH, Bethesda, Maryland, USA.

${ }^{3}$ Wellcome Trust Genome Campus and Sanger Institute, Hinxton, Cambridge, United Kingdom. ${ }^{4}$ Department of Medicine, Massachusetts General Hospital, and Harvard Medical School, Boston, Massachusetts, USA. 5Division of Oral Biology and Department of Physiology and Pharmacology, Canadian Institutes of Health Research (CIHR) Group in Skeletal Development and Remodeling, Schulich School of Medicine and Dentistry, University of Western Ontario, London, Ontario, Canada. ${ }^{6}$ Division of Rheumatology, Northwestern University Feinberg School of Medicine, Chicago, Illinois, USA. IInstitute of Cardiovascular Sciences, St. Boniface General Hospital Research Centre, Winnipeg, Manitoba, Canada. ${ }^{8}$ Institute of Circulatory and Respiratory Health, Canadian Institutes of Health Research, Ottawa, Ontario, Canada.

\begin{abstract}
Chromatin remodeling, particularly histone acetylation, plays a critical role in the progression of pathological cardiac hypertrophy and heart failure. We hypothesized that curcumin, a natural polyphenolic compound abundant in the spice turmeric and a known suppressor of histone acetylation, would suppress cardiac hypertrophy through the disruption of $\mathrm{p} 300$ histone acetyltransferase-dependent (p300-HAT-dependent) transcriptional activation. We tested this hypothesis using primary cultured rat cardiac myocytes and fibroblasts as well as two well-established mouse models of cardiac hypertrophy. Curcumin blocked phenylephrin-induced (PEinduced) cardiac hypertrophy in vitro in a dose-dependent manner. Furthermore, curcumin both prevented and reversed mouse cardiac hypertrophy induced by aortic banding (AB) and PE infusion, as assessed by heart weight/BW and lung weight/BW ratios, echocardiographic parameters, and gene expression of hypertrophic markers. Further investigation demonstrated that curcumin abrogated histone acetylation, GATA4 acetylation, and DNA-binding activity through blocking P300-HAT activity. Curcumin also blocked AB-induced inflammation and fibrosis through disrupting $\mathrm{p} 300-\mathrm{HAT}$-dependent signaling pathways. Our results indicate that curcumin has the potential to protect against cardiac hypertrophy, inflammation, and fibrosis through suppression of p300-HAT activity and downstream GATA4, NF- $\kappa B$, and TGF- $\beta-S m a d$ signaling pathways.
\end{abstract}

\section{Introduction}

Cardiac hypertrophy is an adaptive enlargement of the myocardium in response to increased workload, characterized by an increase in the size of individual cardiac myocytes and whole-organ enlargement. Although cardiac hypertrophy may initially be compensatory, sustained pathologic hypertrophy is deleterious and may lead to heart failure, sudden death, and stroke (1-3). Histone acetylation is one of the key control points for gene regulation in the hypertrophic myocardium (4). Acetylation of histone tails, mediated by histone acetyltransferases (HATs), confers accessibility of the DNA template to the transcriptional machinery and is associated with activation of gene expression (5). Histone deacetylases (HDACs), on the other hand, catalyze removal of acetyl groups on aminoterminal lysine residues of histones and, by promoting nucleosomal condensation, act as transcriptional repressors or silencers of genes (5). The status of histone acetylation is therefore determined by the balanced action of HATs and HDACs. p300 is a critical HAT

Nonstandard abbreviations used: $\mathrm{AB}$, aortic banding; $\mathrm{ANP}$, atrial natriuretic peptide; BNP, brain natriuretic peptide; CTGF, connective tissue growth factor; FS fractional shortening: HAT, histone acetyltransferase; HDAC, histone deacetylase; $\mathrm{HW}$, heart weight; IKK, IKB kinase; LDH, lactate dehydrogenase; LVEDD, LV enddiastolic diameter; LVESD, LV end-systolic diameter; LW, lung weight; MHC, myosin heavy chain; MPO, myeloperoxidase; PE, phenylephrin; PSR, picrosirius red; WGA, wheat germ agglutinin.

Conflict of interest: The authors have declared that no conflict of interest exists. Citation for this article: J. Clin. Invest. 118:879-893 (2008). doi:10.1172/JCI32865. in muscle that modifies chromatin and associated transcription factors and promotes gene activation $(6,7)$. Recent studies have demonstrated that p300 transcriptional activity is enhanced during agonist-induced cardiac hypertrophy and that subsequent blocking of p300-HAT activity inhibits agonist-mediated cardiac growth $(6,8)$. Moreover, transgenic mice that overexpress p300 in the heart develop cardiac hypertrophy and eventual heart failure (9). Therefore, p300-HAT is an attractive target to treat or prevent cardiac hypertrophy and subsequent heart failure.

Curcumin is a natural polyphenolic compound abundant in the rhizome of the perennial herb turmeric, Curcuma longa (10). It is commonly used as a dietary spice and coloring agent in cooking and is used anecdotally as an herb in traditional Indian and Chinese medicine (11). However, to our knowledge no study to date has addressed the effect of curcumin on cardiac hypertrophy and related signaling pathways. Although evidence demonstrates that curcumin is an inhibitor of p300-HAT $(12,13)$, very little is known about whether this regulatory effect is related to a protective role cardiac dysfunction. Therefore, we aimed to determine whether curcumin attenuates cardiac hypertrophy in vitro and in vivo by impairing p300-HAT activity.

\section{Results}

Pretreatment with curcumin in bibits cardiac bypertrophy in vitro. Neonatal rat ventricular myocytes were cultured to greater than $97 \%$ purity, as confirmed by phase-contrast microscopy and 
A

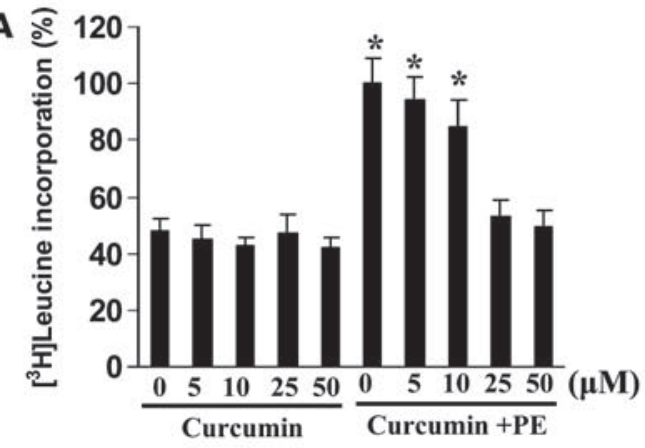

B

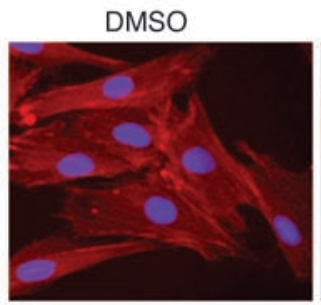

C

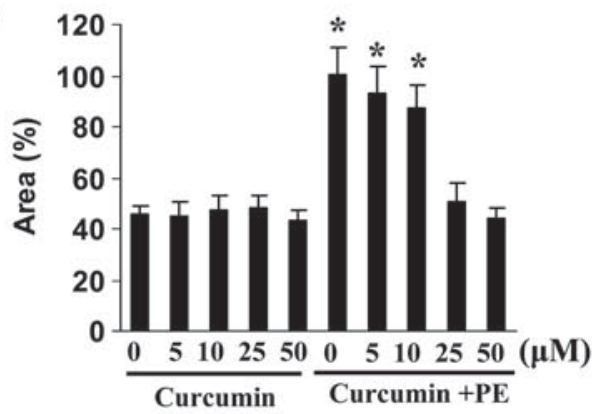

D

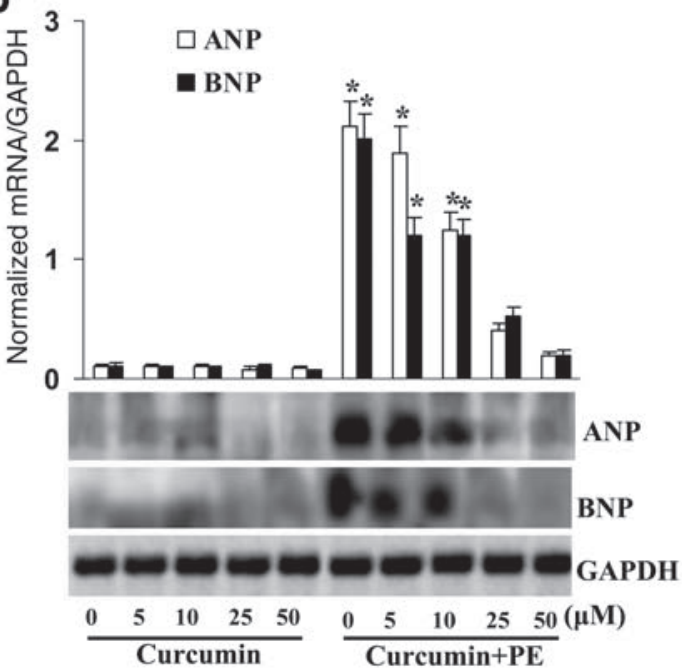

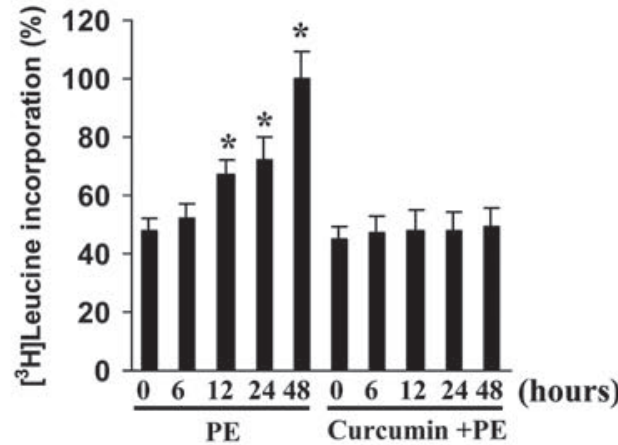

PE

$\mathrm{PE}+$ Curcumin
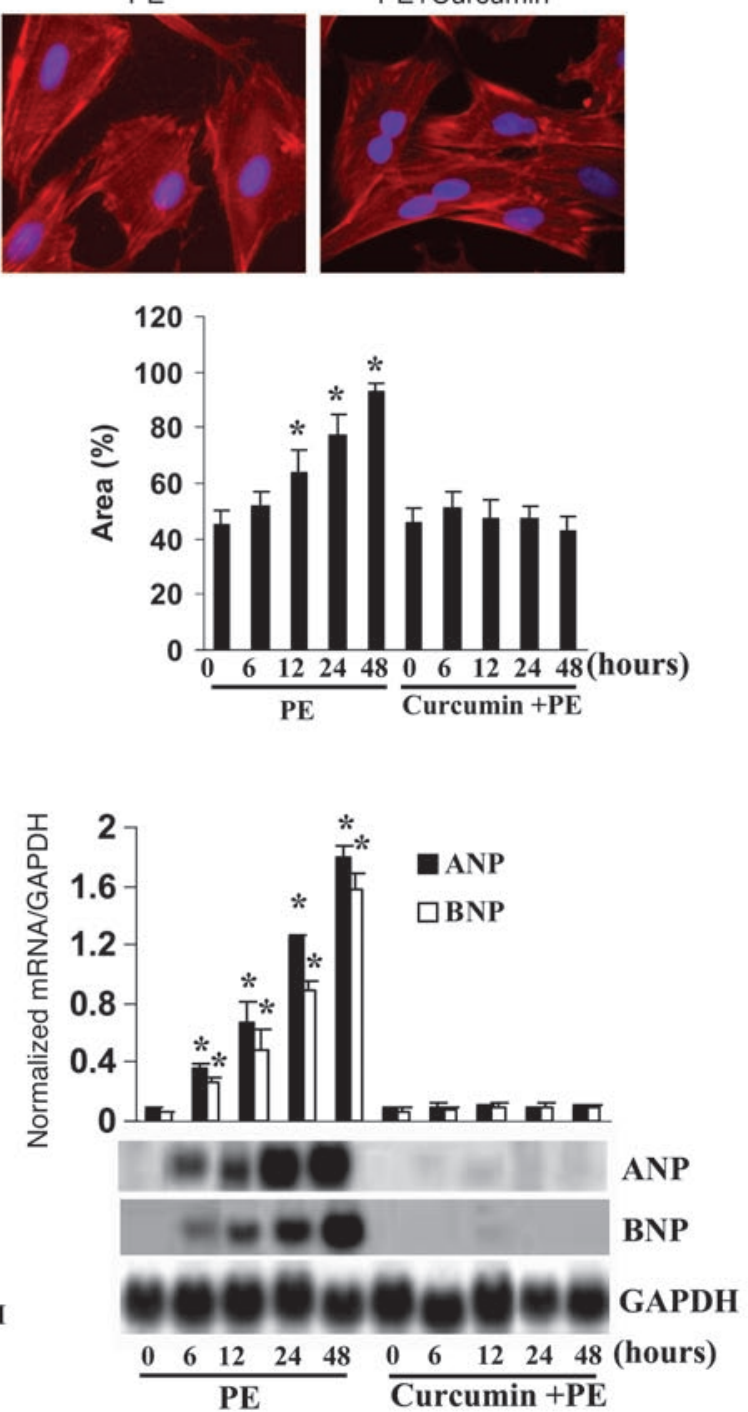

Figure 1

Pretreatment with curcumin inhibits cardiac hypertrophy in vitro. (A) Curcumin inhibited PE-induced $\left[{ }^{3} \mathrm{H}\right]$ leucine incorporation. (B) Representative fields of cardiac myocytes stained with $\alpha$-actinin. Original magnification, $\times 200$. (C) Quantification of cell cross-sectional area from experiments shown in B by measuring 50 random cells. (D) Curcumin blunted PE-induced ANP and BNP mRNA expression levels by Northern blot. $\left.{ }^{3} \mathrm{H}\right]$ leucine incorporation and Northern blot were measured as described in Methods. The results were reproducible in 3 separate experiments. ${ }^{\star} P<0.05$ versus control. 
immunocytochemical staining. To rule out the possibility of cytotoxicity, we determined the number of viable cells in all wells using trypan blue exclusion analysis and lactate dehydrogenase $(\mathrm{LDH})$ release assay. When 5-50 $\mu \mathrm{M}$ curcumin was applied to cultured neonatal cardiomyocytes, cells were observed to be healthy even in the presence of $50 \mu \mathrm{M}$ curcumin at the end of 48 hours (Supplemental Figure 1; supplemental material available online with this article; doi:10.1172/JCI32865DS1). At $100 \mu \mathrm{M}$ curcumin, however, we observed decreased cell viability, leading us to choose a lower dose for the in vitro experiments. There were no observable adverse effects by the administration of DMSO and phenylephrin (PE) or infection with a wild-type human p300 cDNA adenoviral (Ad-p300) and an AT2 mutant p300 adenoviral lacking HAT activity (Ad-DN-p300) (data not shown). In this study, curcumin was dissolved in DMSO medium for the in vitro studies. DMSO alone without curcumin served as a control and did not show any effect on cell viability, cardiac hypertrophy, collagen synthesis, and related molecular mechanisms (data not shown).

Cardiac hypertrophy can be monitored by increased protein synthesis, myocyte cross-sectional area, and induction of fetal gene expression (14). Cardiac myocytes were incubated with curcumin for 60 minutes and subsequently treated with $100 \mu \mathrm{M}$ PE for 48 hours. Pretreatment with curcumin demonstrated a dose-dependent reduction in PE-induced increases of $\left[{ }^{3} \mathrm{H}\right]$ leucine incorporation that showed maximal effects at $50 \mu \mathrm{M}$ (Figure 1A). Additionally, the increase in cardiac myocyte size seen after 48 hours of culture in the presence of $\mathrm{PE}$ was also markedly attenuated by curcumin (Figure 1, B and C). Curcumin markedly reduced atrial natriuretic peptide (ANP) and brain natriuretic peptide (BNP) mRNA expression levels induced by PE (Figure 1D). The inhibition of cardiac hypertrophy in vitro by curcumin was sustained for all tested times. However, curcumin alone had no effect on $\left[{ }^{3} \mathrm{H}\right]$ leucine incorporation, cardiac myocyte size, or expression of ANP and BNP. These data demonstrate that curcumin attenuates cardiac hypertrophy in vitro.

Pretreatment with curcumin inhibits cardiac bypertrophy in vivo. To determine the physiological relevance of our in vitro findings, we investigated the effects of curcumin in a murine pressure-overload model of cardiac hypertrophy. To evaluate the dose-response relationship, we administered 3 different doses of curcumin (50, 75 , and $100 \mathrm{mg} / \mathrm{kg} / \mathrm{d}$ ) for 1 week and then subjected the mice to either chronic pressure overload generated by aortic banding (AB) or sham surgery (control). We found that maximal efficacy was achieved at a curcumin dose of $75 \mathrm{mg} / \mathrm{kg} / \mathrm{d}$ (Supplemental Table 1). Moreover, no apparent effect on cell toxicity was observed with any dose of curcumin, and $75 \mathrm{mg} / \mathrm{kg} / \mathrm{d}$ was therefore chosen as the experimental dose. These findings are in agreement with previous publications in which curcumin showed no toxicity in the liver or kidney at doses of 100 or $200 \mathrm{mg} / \mathrm{kg} / \mathrm{d}(15,16)$.

In order to further evaluate the effects of curcumin on cardiac hypertrophy, mice were randomly assigned to 4 groups: pretreatment with either vehicle or $75 \mathrm{mg} / \mathrm{kg} / \mathrm{d}$ curcumin for 1 week prior to either $A B$ surgery or sham operation. Curcumin treatment of the $\mathrm{AB}$ mice resulted in significant attenuation of hypertrophy, as measured by heart weight/BW (HW/BW) ratio, lung weight/BW $(\mathrm{LW} / \mathrm{BW})$ ratio, and cardiomyocyte cross-sectional area (Figure 2A). No significant changes were observed in the sham-operated mice treated with curcumin or vehicle. Gross heart and wheat germ agglutinin (WGA) staining further confirmed the inhibitory effect of curcumin on cardiac remodeling in $\mathrm{AB}$ hearts (Figure $2 \mathrm{~B}$ ). As shown in Table 1, curcumin pretreatment prevented adverse cardiac remodeling and ventricular dysfunction, as evidenced by improvements in LV end-systolic diameter (LVESD), LV end-diastolic diameter (LVEDD), and percent fractional shortening (FS).

We next examined the potential effect of curcumin on hypertrophy mediated by PE infusion. Mice were randomly allocated into 4 groups: pretreatment with either vehicle or $75 \mathrm{mg} / \mathrm{kg} / \mathrm{d}$ curcumin for 1 week prior to either PE or saline infusion. Osmotic minipumps were implanted subcutaneously for a 3-week administration period followed by cardiac functional assessment. As shown in Table 2, curcumin abrogated PE-induced cardiac chamber dilation in both systole and diastole. The PE-induced increase in HW/BW and LW/BW ratios as well as cardiomyocyte cross-sectional area were also attenuated by 4 weeks of curcumin administration (Figure 2C). These findings were confirmed by morphological assessment (Figure 2D).

ANP, BNP, and myosin heavy chain $\beta$ ( $\beta$-MHC) are markers for cardiac hypertrophy (14). To determine whether curcumin affected the mRNA expression levels of these markers, we performed Northern blot analysis. Curcumin attenuated the observed increase in hypertrophic marker expression caused by AB or PE infusion (Figure 2, $\mathrm{E}$ and $\mathrm{F}$ ). These findings suggest that curcumin prevents the development of cardiac hypertrophy in vivo.

Pretreatment with curcumin inbibits bistone acetylation in response to bypertrophic stimuli. To explore the molecular mechanisms through which curcumin impairs the hypertrophic response, we examined the state of acetylation of histones by assaying the incorporation of $\left[{ }^{3} \mathrm{H}\right]$ acetate into histones. We exposed cultured neonatal rat cardiomyocytes to $100 \mu \mathrm{M}$ PE with or without curcumin. As expected, $\mathrm{PE}$ induced a significant increase in histone acetylation that was dose-dependently blocked and sustained for all tested time points by curcumin (Figure 3, A and B). These findings were confirmed by concomitant attenuation of histone $\mathrm{H} 3$, histone $\mathrm{H} 4$, and tubulin acetylation (Figure 3C). To test its efficacy in vivo, Western blot analysis was performed using samples from the 2 differing hypertrophic animal models. Similar findings confirmed that vehicle-treated $\mathrm{AB}$ or PE-infused mice markedly induced the acetylation of histone $\mathrm{H} 3$, histone $\mathrm{H} 4$, and tubulin and that curcumin-treated mice significantly attenuated the acetylation of these targets (Figure 3, D and E). Our findings suggest that curcumin inhibits histone acetylation in vitro and in vivo in response to hypertrophic stimuli.

Pretreatment with curcumin blocks p300-HAT activity, but not HDAC activity. Histone acetylation is regulated by p300-HAT and plays an important role in heart disease (9). To elucidate the role of p300 in the inhibitory effect of curcumin on cardiac hypertrophy, we analyzed the effects of curcumin on p300-HAT activity induced by PE. As shown in Figure 4A, at concentrations of 50 and $100 \mu \mathrm{M}$ PE, p300-HAT activity increased 11.3- and 17.9-fold, respectively, in cardiac myocytes. The linear increase of HAT activity by increased concentrations of PE indicated that PE-induced P300-HAT activity was dose dependent and maximally achieved at $100 \mu \mathrm{M}$. p300-HAT activity reached its maximum value at 6 hours after the addition of $100 \mu \mathrm{M}$ PE (Figure 4B). We also found that curcumin dosedependently blocked PE-induced p300-HAT activity and sustained this inhibition for all tested time points (Figure 4, C and D). We further demonstrated that blocking p300-HAT activity inhibited, whereas increased p300-HAT activity promoted, histone acetylation induced by PE, as estimated by the global acetylation of histones and the acetylation of histone $\mathrm{H} 3$, histone $\mathrm{H} 4$, and tubulin (Figure 4, E and F). This suggests that the hyperacetylation induced by PE in cardiac myocytes is dependent on p300-HAT activity. To define 
A

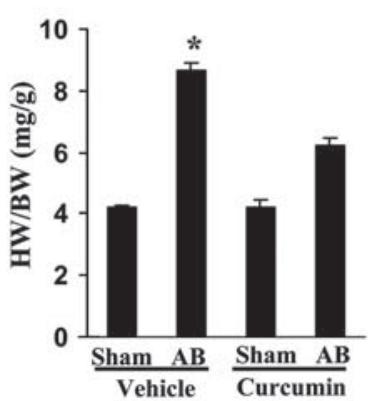

B

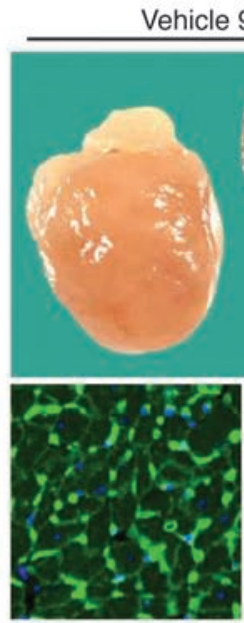

Sham

C

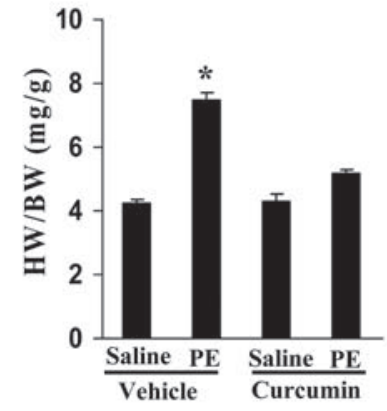

D

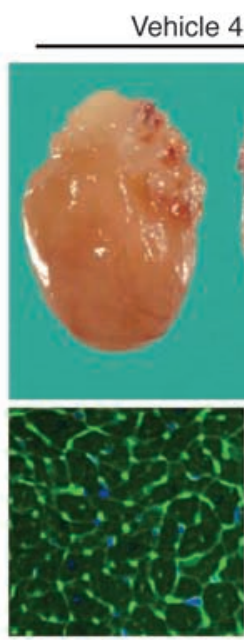

Saline

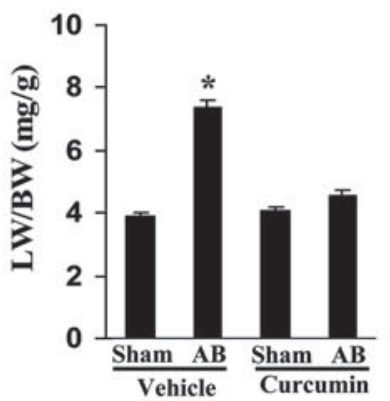

Curcumin 9 weeks

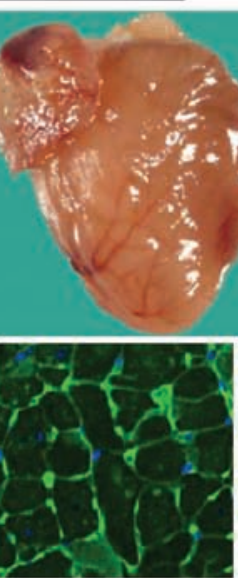

AB 8 weeks

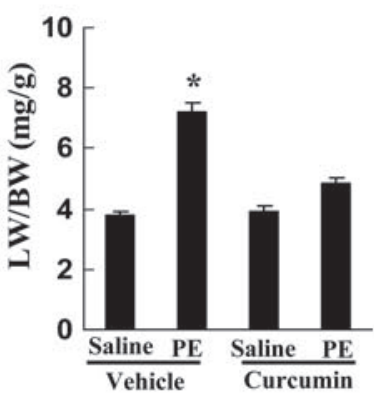

Curcumin 4 weeks

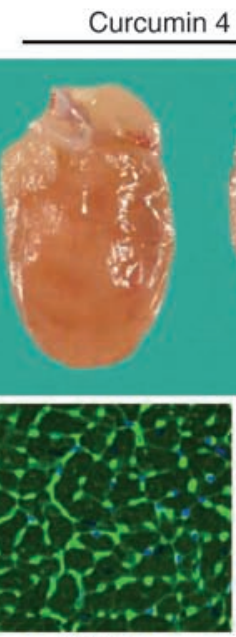

Saline
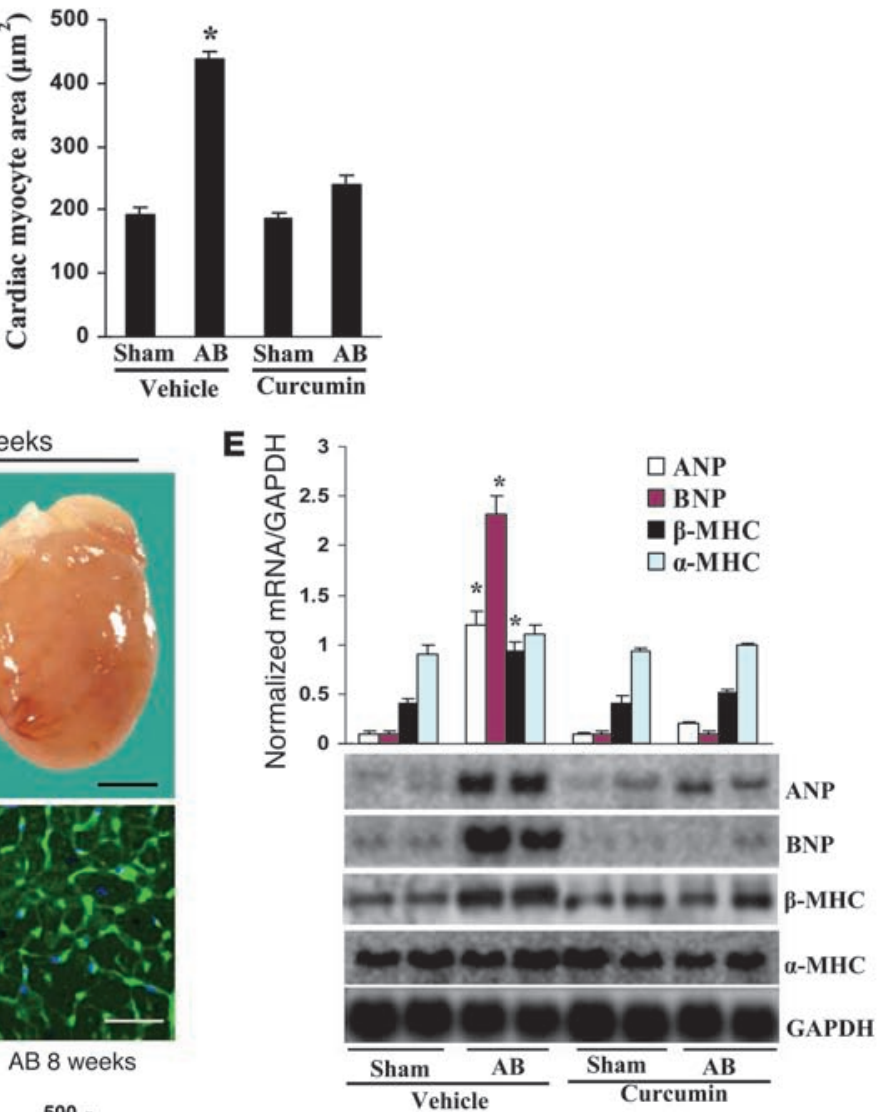

AB 8 weeks

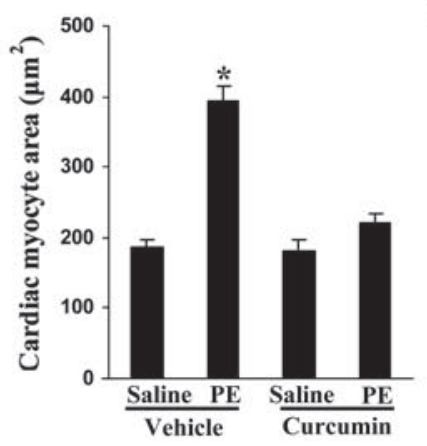

Vehicle
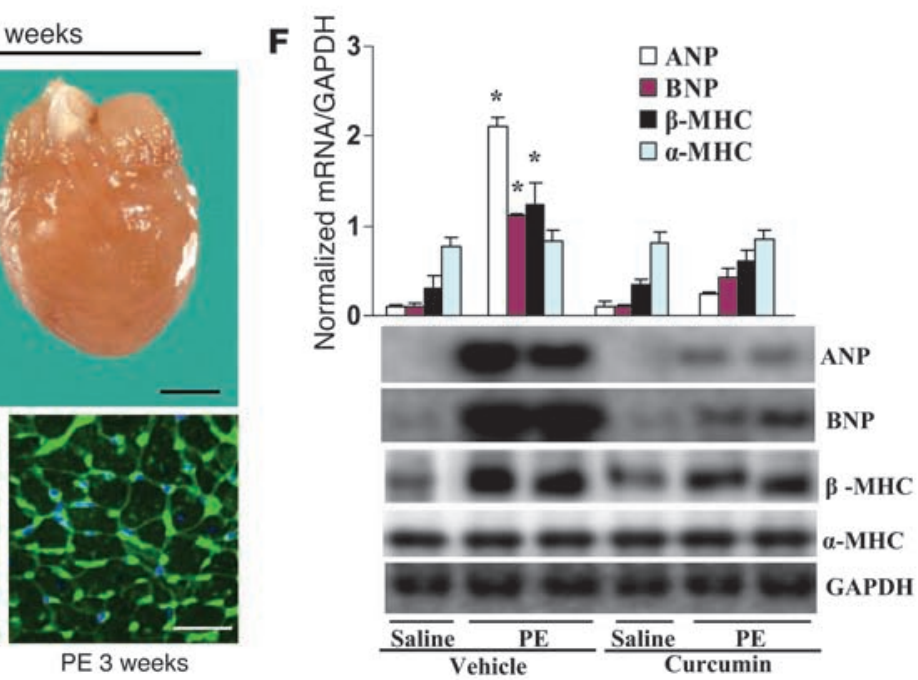

PE 3 weeks

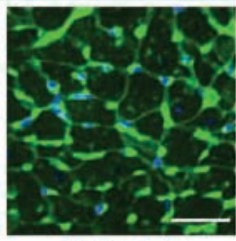




\section{Figure 2}

Pretreatment with curcumin blunts cardiac hypertrophy in vivo. (A and C) Statistical results of HW/BW ratio, LW/BW ratio, and myocyte crosssectional areas ( $n=200$ cells per section) at 8 weeks after AB surgery $(\mathbf{A} ; n=6)$ or 3 weeks after PE infusion (C; $n=7)$. (B and D) Gross heart and WGA-FITC staining of sham and $A B$ mice at 8 weeks after surgery (B) or 3 weeks of saline- and PE-infused mice (D) treated with or without curcumin. Scale bars: $20 \mathrm{~mm}$ (gross heart); $50 \mu \mathrm{m}$ (WGA stain). (E and F) Analysis of hypertrophic markers $(n=4)$. Total RNA was isolated from hearts of mice of the indicated groups, and expression of transcripts for ANP, BNP, $\beta-\mathrm{MHC}$, and $\alpha-\mathrm{MHC}$ induced by $A B(E)$ or $P E$ infusion $(F)$ was determined by Northern blot analysis. ${ }^{\star} P<0.05$ versus respective vehicle control.

the role of HDAC activity in curcumin-induced histone hypoacetylation, the effect of curcumin on HDAC activity was determined. Curcumin did not alter its activity at each of the tested concentrations (Supplemental Figure 2). Collectively, these results indicate that curcumin inhibits histone acetylation through direct inhibition of p300-HAT activity rather than HDAC activity.

Pretreatment with curcumin blocks GATA4 acetylation and DNAbinding activity. p300 protein serves as an adaptor for hypertrophy-responsive transcription factors including GATA4, which is required for the activation of cardiac genes that are upregulated during cardiac hypertrophy $(17,18)$. As expected, both PE stimulation and $\mathrm{AB}$ markedly induced, and curcumin pretreatment completely abolished, GATA4 acetylation and DNA-binding activity (Figure 5, A and B). Based on these observed inhibitory effects, we investigated whether PE-mediated GATA4 activation is dependent on p300-HAT activity. Our results demonstrated that PE-induced GATA4 acetylation and DNA-binding activity were almost completely inhibited by infection with Ad-DN-p300 but were augmented by infection with Ad-p300 (Figure 5C). In addition, the inhibitory effects of curcumin on GATA4 acetylation and DNAbinding activity were reversed by infection with Ad-p300 (Figure 5D). These data suggest that GATA4 acetylation and activation by PE require p300-HAT activity in cardiac myocytes.

Pretreatment with curcumin blunts inflammation and fibrosis. Inflammation is known to play an important role in the development and progression to hypertrophy and heart failure (19-21). To determine whether curcumin suppresses inflammation in the heart, we first examined the cellular infiltrates by immunostaining analyses. As shown in Figure 6A, the population of cells that stained posi- tive for myeloperoxidase (MPO), Mac-1, and Mac-3 significantly increased at 8 weeks after $\mathrm{AB}$; these increases were dramatically reduced by curcumin treatment. Furthermore, curcumin decreased NF- $\kappa \mathrm{B}$ activation and MCP-1, IL-6, IL- $1 \beta$, and TNF- $\alpha$ mRNA and protein expression induced by $\mathrm{AB}$ (Supplemental Figure 3 , A and $\mathrm{B}$, and Figure 6B). Curcumin treatment also impaired IкB $\alpha$ phosphorylation and degradation, IКB kinase (IKK) activation, and p65 translocation mediated by AB (Supplemental Figure 3, C-F). A previous report describing the regulation of NF- $\mathrm{KB}$ activation by direct protein-protein interaction with p300 (22) prompted us to investigate the functional significance of p300 on NF-кB signaling. Infection with Ad-p300 promoted, and infection with Ad-DN-p300 downregulated, NF-KB transcription and cytokine expression after PE treatment in myocytes (Supplemental Figure 3, $\mathrm{G}$ and $\mathrm{H}$ ). Furthermore, $\mathrm{p} 300$ overexpression significantly reversed the curcumin-induced inhibitory effects on inflammation (Supplemental Figure 3I). These results indicate that curcumin blocks $\mathrm{NF}-\kappa \mathrm{B}$ signaling and NF- $\mathrm{KB}-$ dependent inflammatory responses through the disruption of p300-HAT activity.

Pathological cardiac hypertrophy is associated with increased fibrosis in the myocardium (1), and, as expected, marked perivascular and interstitial fibrosis were detected in the vehicle-treated $\mathrm{AB}$ mice by picrosirius red (PSR) staining. Curcumin treatment, however, remarkably reduced the extent of cardiac fibrosis in vivo (Figure 6, C and D). Subsequent analysis of mRNA and protein expression levels of known mediators of fibrosis including TGF- $\beta 1$, collagen I, and connective tissue growth factor (CTGF) demonstrated a blunted response by curcumin treatment (Figure 6, E and F). In addition, curcumin effectively blocked collagen synthesis and COL1A2, PAI-1, and CTGF protein expression levels and promoter activities induced by TGF- $\beta 1$ in cultured cardiac fibroblasts (Supplemental Figure 4, A-C).

To further elucidate the cellular mechanisms underlying the antifibrotic effects of curcumin, we then assessed the regulatory role of curcumin in Smad cascade activation in hearts subjected to AB. Smad-2 phosphorylation and Smad-2/3/4 nuclear translocation were markedly blocked by curcumin (Supplemental Figure 4D). Our in vitro studies using neonatal rat cardiac fibroblasts yielded identical results (Supplemental Figure 4E). To our knowledge, no previous study has shown the physiologic link between TGF- $\beta$ and p300 with respect to collagen synthesis in the heart. Using confluent cardiac fibroblasts infected with Ad-GFP, Ad-p300, or Ad-DN-p300 along with COL1A2-, PAI-1-, or CTGF-luc reporter

\section{Table 1}

Effects of curcumin on cardiac hypertrophy induced by $A B$ over time

\begin{tabular}{|c|c|c|c|c|c|c|c|c|}
\hline \multirow[b]{2}{*}{ Group (n) } & \multicolumn{4}{|c|}{ Vehicle } & \multicolumn{4}{|c|}{ Curcumin, $75 \mathrm{mg} / \mathrm{kg} / \mathrm{d}$} \\
\hline & Basal (12) & $A B, 4$ wk (10) & $A B, 6$ wk (8) & $A B, 8$ wk (8) & Basal (12) & AB, 4 wk (11) & $A B, 6$ wk (7) & $A B, 8$ wk (8) \\
\hline $\mathrm{BW}(\mathrm{g})$ & $24.2 \pm 1.3$ & $25.0 \pm 2.1$ & $26.3 \pm 1.4$ & $28.0 \pm 1.2$ & $24.6 \pm 2.0$ & $26.4 \pm 2.3$ & $26.8 \pm 1.7$ & $28.3 \pm 1.3$ \\
\hline $\mathrm{SBP}(\mathrm{mmHg})$ & $105.0 \pm 3.7$ & $137.6 \pm 5.7^{A}$ & $158.0 \pm 9.6^{A}$ & $163.0 \pm 3.9^{A}$ & $108.0 \pm 3.8$ & $136.6 \pm 6.5^{\mathrm{A}}$ & $155.7 \pm 7.1^{\mathrm{A}}$ & $161.4 \pm 6.2^{\mathrm{A}}$ \\
\hline $\mathrm{HR}(\mathrm{bpm})$ & $445 \pm 3$ & $446 \pm 22$ & $452 \pm 24$ & $454 \pm 23$ & $441 \pm 36$ & $445 \pm 26$ & $459 \pm 17$ & $436 \pm 25$ \\
\hline LVEDD (mm) & $3.65 \pm 0.03$ & $4.12 \pm 0.02^{\mathrm{A}}$ & $4.89 \pm 0.05^{A}$ & $5.22 \pm 0.08^{A}$ & $3.69 \pm 0.06$ & $3.72 \pm 0.05^{B}$ & $3.81 \pm 0.06^{B}$ & $4.02 \pm 0.03^{B}$ \\
\hline LVESD (mm) & $1.79 \pm 0.02$ & $2.52 \pm 0.04^{\mathrm{A}}$ & $3.22 \pm 0.06^{A}$ & $3.81 \pm 0.04^{A}$ & $1.70 \pm 0.03$ & $1.78 \pm 0.03^{B}$ & $1.96 \pm 0.06^{B}$ & $2.09 \pm 0.04 \mathrm{~B}$ \\
\hline IVSd (mm) & $0.64 \pm 0.06$ & $0.92 \pm 0.08$ & $1.25 \pm 0.07^{A}$ & $1.62 \pm 0.04^{A}$ & $0.67 \pm 0.02$ & $0.73 \pm 0.02$ & $0.81 \pm 0.07^{B}$ & $0.88 \pm 0.07^{B}$ \\
\hline LVPWd (mm) & $0.67 \pm 0.07$ & $0.78 \pm 0.04$ & $1.19 \pm 0.05^{A}$ & $1.36 \pm 0.08^{A}$ & $0.60 \pm 0.03$ & $0.71 \pm 0.05$ & $0.78 \pm 0.03^{B}$ & $0.82 \pm 0.02^{B}$ \\
\hline $\mathrm{FS}(\%)$ & $55.4 \pm 3.1$ & $43.0 \pm 2.3^{\mathrm{A}}$ & $30.0 \pm 1.7^{\mathrm{A}}$ & $18.2 \pm 4.2^{\mathrm{A}}$ & $56.8 \pm 4.3$ & $50.7 \pm 2.9^{A, B}$ & $47.9 \pm 1.5^{\mathrm{A}, \mathrm{B}}$ & $40.7 \pm 2.5^{\mathrm{A}, \mathrm{B}}$ \\
\hline
\end{tabular}

All values are mean \pm SEM. SBP, systolic blood pressure; HR, heart rate; IVSd, LV septum, diastolic; LVPWd, LV posterior wall, diastolic. AP < 0.05 versus sham. ${ }^{B} P<0.05$ versus vehicle after $A B$. 
Table 2

Effects of curcumin on cardiac hypertrophy induced by PE infusion

\begin{tabular}{|c|c|c|c|c|c|c|}
\hline \multirow[b]{2}{*}{ Group (n) } & \multicolumn{3}{|c|}{ Vehicle } & \multicolumn{3}{|c|}{ Curcumin, $75 \mathrm{mg} / \mathrm{kg} / \mathrm{d}$} \\
\hline & Basal (15) & Saline (13) & PE, 3 wk (8) & Saline (14) & $\begin{array}{c}\text { PE, } 3 \text { wk + } \\
\text { Cur, } 4 \text { wk (10) }\end{array}$ & $\begin{array}{c}\text { PE, } 3 \text { wk + } \\
\text { Cur, } 2 \text { wk (8) }\end{array}$ \\
\hline BW (g) & $26.2 \pm 2.2$ & $27.8 \pm 2.3$ & $26.4 \pm 1.8$ & $27.5 \pm 1.4$ & $27.4 \pm 2.1$ & $27.0 \pm 1.7$ \\
\hline $\mathrm{SBP}(\mathrm{mmHg})$ & $110.5 \pm 2.1$ & $113.0 \pm 4.2$ & $159.5 \pm 3.6^{\mathrm{A}}$ & $104.2 \pm 3.2$ & $153.0 \pm 4.1^{\mathrm{A}}$ & $155.7 \pm 3.9^{A}$ \\
\hline $\mathrm{HR}$ (bpm) & $488 \pm 18$ & $465 \pm 15$ & $481 \pm 21$ & $475 \pm 34$ & $467 \pm 49$ & $464 \pm 26$ \\
\hline LVEDD (mm) & $3.81 \pm 0.03$ & $3.82 \pm 0.05$ & $5.16 \pm 0.02^{A}$ & $3.74 \pm 0.05$ & $3.98 \pm 0.01^{B}$ & $4.20 \pm 0.02^{A, B}$ \\
\hline $\operatorname{LVESD}(\mathrm{mm})$ & $1.67 \pm 0.02$ & $1.73 \pm 0.03$ & $3.78 \pm 0.02^{A}$ & $1.59 \pm 0.01$ & $1.88 \pm 0.01^{B}$ & $2.14 \pm 0.01^{\mathrm{A}, \mathrm{B}}$ \\
\hline IVSd (mm) & $0.64 \pm 0.02$ & $0.73 \pm 0.04$ & $1.35 \pm 0.01^{A}$ & $0.58 \pm 0.03$ & $0.83 \pm 0.02^{B}$ & $0.87 \pm 0.02^{B}$ \\
\hline LVPWd (mm) & $0.61 \pm 0.01$ & $0.62 \pm 0.02$ & $1.21 \pm 0.02^{\mathrm{A}}$ & $0.63 \pm 0.01$ & $0.66 \pm 0.03^{B}$ & $0.72 \pm 0.03^{B}$ \\
\hline FS (\%) & $56.0 \pm 1.3$ & $55.0 \pm 2.2$ & $25.3 \pm 2.1^{\mathrm{A}}$ & $54.3 \pm 1.6$ & $45.0 \pm 1.3^{A, B}$ & $39.7 \pm 1.7^{\mathrm{A}, \mathrm{B}}$ \\
\hline
\end{tabular}

All values are mean \pm SEM. Cur, curcumin; SBP, systolic blood pressure; HR, heart rate; IVSd, LV septum, diastolic; LVPWd, LV posterior wall, diastolic. ${ }^{A} P<0.05$ versus saline. ${ }^{B} P<0.05$ versus vehicle after $P E$ infusion.

constructs, we incubated the cells with TGF- $\beta 1$. Forced expression of ectopic p300 revealed a significant increase in, whereas inhibiting p300-HAT activity almost completely abrogated, collagen synthesis, promoter activities, and protein levels of markers of fibrosis (Supplemental Figure 4F). Immunoblot analysis further demonstrated that Ad-DN-p300 almost completely abrogated the phosphorylation of Smad-2 and nuclear translocation of Smad-2/3/4 in response to TGF- $\beta 1$ (Supplemental Figure 4G). Ad-p300 infection partially rescued the curcumin-induced inhibitory effects on collagen synthesis, markers of fibrosis, phosphorylation of Smad-2, and translocation of Smad-2/3/4 (Supplemental Figure $4, \mathrm{H}$ and I). These findings suggest that curcumin blocks

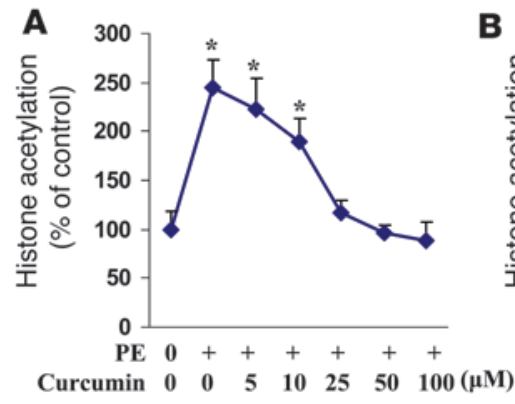

D

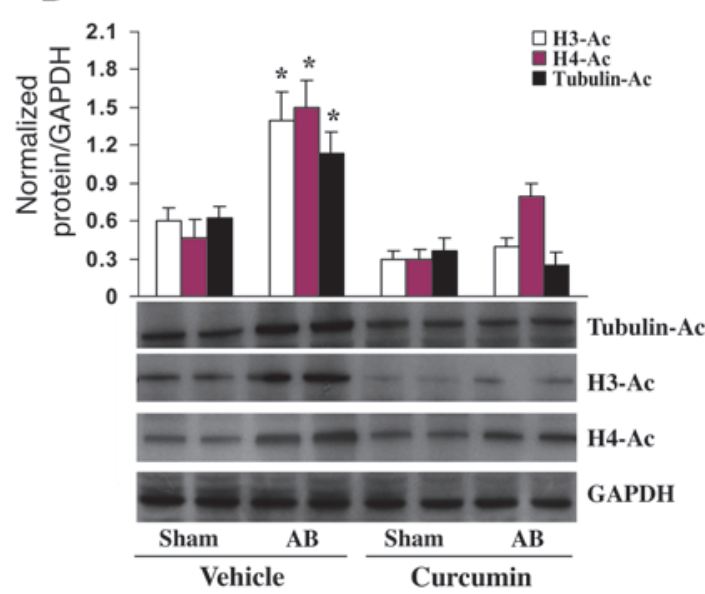

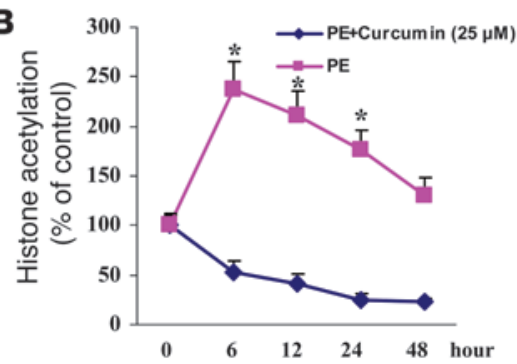
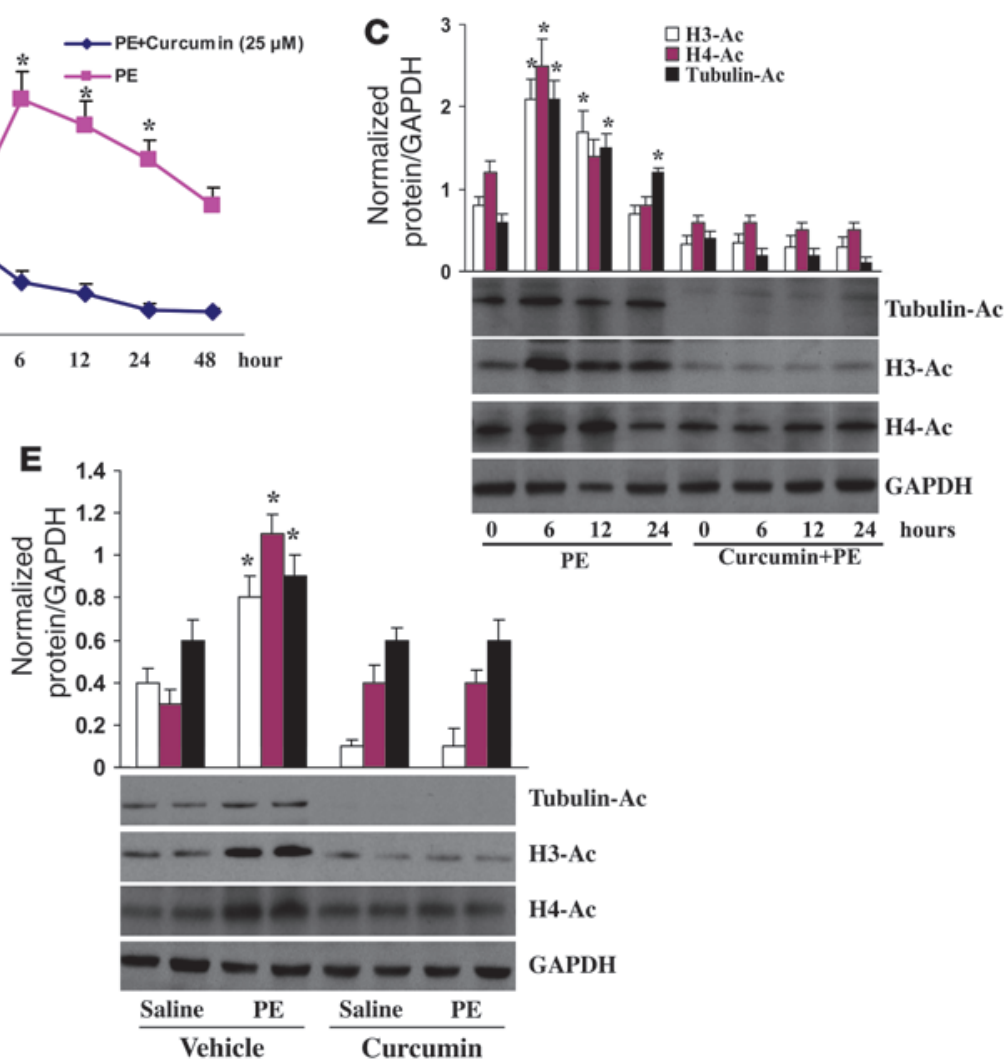

Figure 3

Pretreatment with curcumin inhibits histone acetylation in vitro and in vivo. (A and $\mathbf{B}$ ) The dose and time courses of curcumin on the global acetylation of histones induced by PE. The results of 4 parallel experiments are shown. (C) Curcumin inhibited the acetylation of histone $\mathrm{H} 3$, histone $\mathrm{H} 4$, and tubulin. Experiments were performed in triplicate. The levels of histone $\mathrm{H} 3$ acetylation, histone $\mathrm{H} 4$ acetylation, and tubulin were quantified and normalized relative to GAPDH. ${ }^{*} P<0.05$ versus control. (D and E) Curcumin blocked AB-and PE infusion-mediated acetylation of histone $\mathrm{H} 3$, histone $\mathrm{H} 4$, and tubulin in mice $(n=5)$. Densitometric quantification of acetyl-H3, acetyl-H4, and tubulin was normalized to GAPDH. ${ }^{*} P<0.05$ versus respective vehicle control. 
A

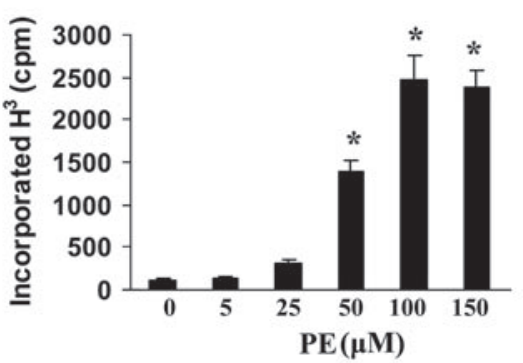

C

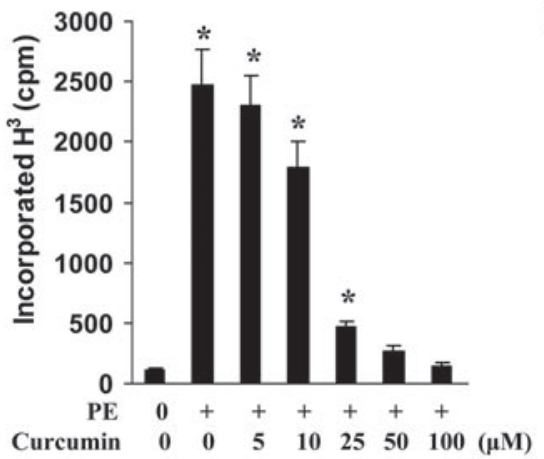

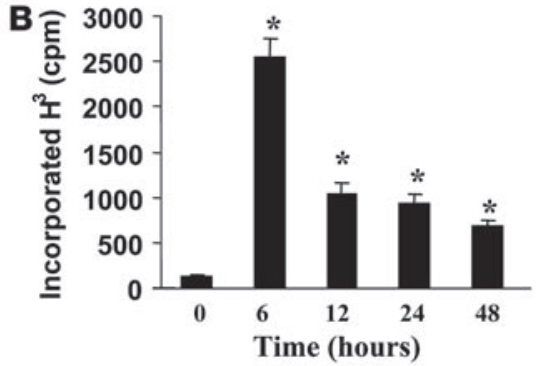

D

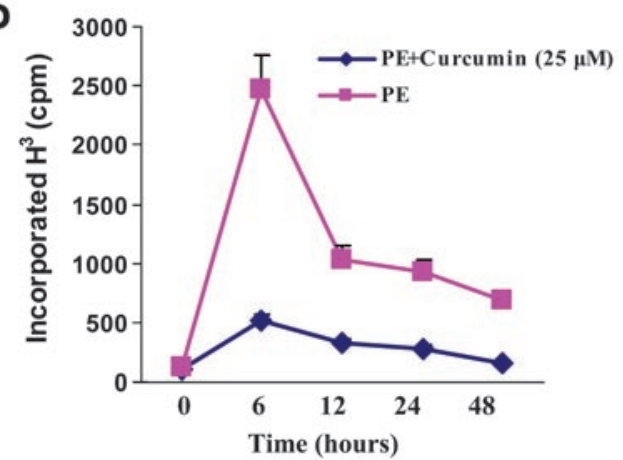

E

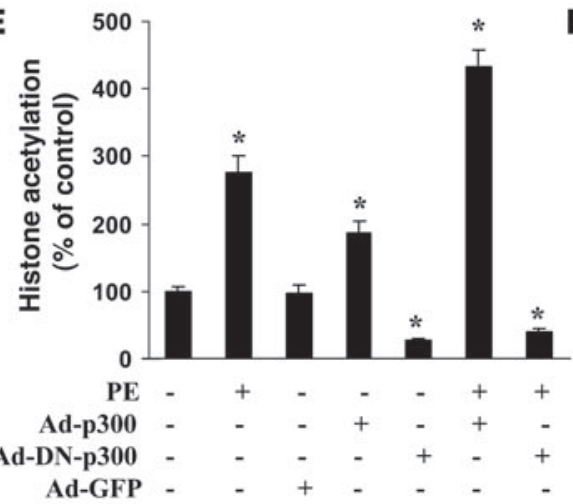

$\mathbf{F}$

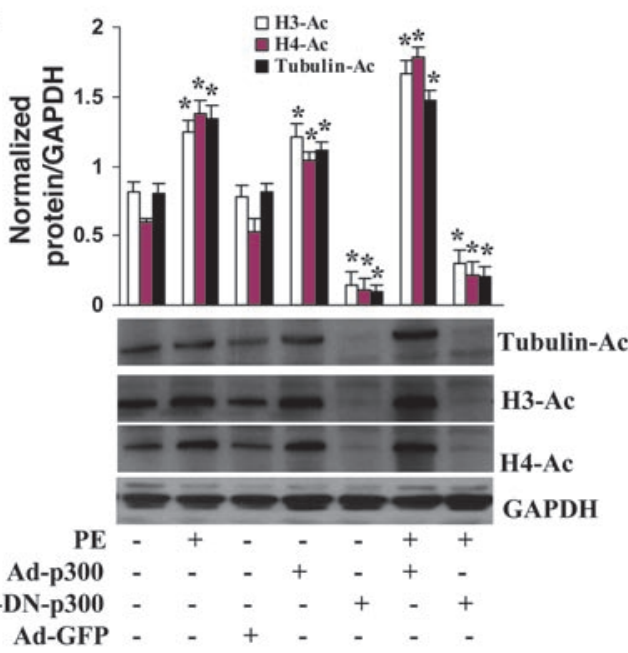

Figure 4

Pretreatment with curcumin blocks p300-HAT activity. (A and B) The dose and time course of PE on HAT activity of p300. Cells were treated with different doses of PE $(\mathbf{A})$ or with $100 \mu \mathrm{M}$ PE for the indicated times (B) and then harvested and subjected to analysis of HAT activity as described in Methods. (C and D) Curcumin inhibited PE-induced p300-HAT activity. Cells were either pretreated for 60 minutes with different doses of curcumin and then incubated with 100 $\mu \mathrm{M}$ PE for 6 hours (C) or pretreated for 60 minutes with $25 \mu \mathrm{M}$ curcumin and then incubated with $100 \mu \mathrm{M} \mathrm{PE}$ for different times up to 48 hours (D). (E and F) Effect of p300 on histone acetylation. Cells were infected with Ad-p300, Ad-DN-p300, or Ad-GFP for 24 hours and then treated with $100 \mu \mathrm{M}$ PE for 6 hours. The global acetylation of histones $(\mathbf{E})$ and the acetylation of histone $\mathrm{H} 3$, histone $\mathrm{H} 4$, and tubulin $(\mathbf{F})$ were determined. Each assay was performed at least 3 times. ${ }^{*} P<0.05$ versus control. collagen synthesis by disrupting p300-HAT activity-dependent TGF- $\beta$-Smad signaling.

Curcumin ameliorates established cardiac bypertrophy in vivo. For greater clinical relevance, we next assessed whether curcumin can reverse established cardiac hypertrophy. For these studies, we subjected mice to $A B$ surgery and sham operation (control). Cardiac hypertrophy was confirmed by the increase in HW/BW ratio, by the gross morphology of the heart, and from echocardiographic analyses after a 2-week period. Continuation of $A B$ for a subsequent 6 weeks resulted in the transition to heart failure, as evidenced by a further decline in percent FS, increase in LVEDD and LVESD, and increase in HW/BW and LW/BW ratios (Table 3 and Figure 7, A and B). Interestingly, curcumin treatment for a period of 6 weeks after the initial 2 weeks of $\mathrm{AB}$ reversed the remodeling, contractile dysfunction, and cardiac ANP, BNP, and $\beta$-MHC mRNA expression levels toward normal control values, ultimately preventing the transition to heart failure (Figure 7, A, B, and E). To verify this observation, we tested the impact of curcumin on established PE- induced cardiac hypertrophy. Curcumin was administered to mice at a dose of $75 \mathrm{mg} / \mathrm{kg} / \mathrm{d}$ starting after 1 week of PE infusion and continuing for 2 weeks. Cardiac hypertrophy was markedly reversed by curcumin as assessed by HW/BW and LW/BW ratios, echocardiographic measurements, cardiomyocyte area, and expression levels of hypertrophic markers compared with vehicle-treated controls (Table 2 and Figure 7, C, D and F). These data confirm our findings from the $\mathrm{AB}$ model and suggest that curcumin is able to reverse established cardiac hypertrophy and heart failure.

p300 partially reverses the inhibitory effects of curcumin in vivo. The above experimental results suggested that curcumin inhibits cardiac hypertrophy, inflammation, and fibrosis through blocking p300-HAT-dependent signaling pathways. To confirm these findings, we evaluated whether the inhibitory effects of curcumin could be reversed through ectopic expression of p300 in vivo. Therefore, we established a protocol to locally increase p300 expression via direct adenovirus-mediated gene transfer into the heart. Western blot analysis showed that p300 protein levels were significantly 


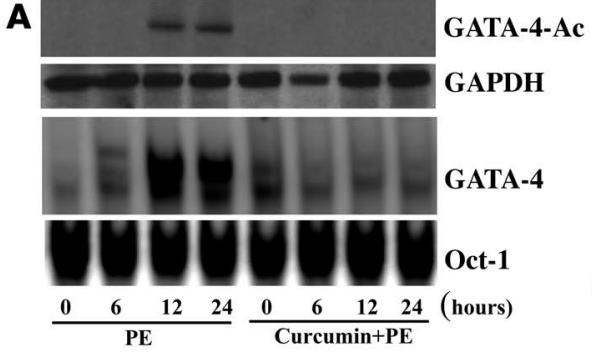

B

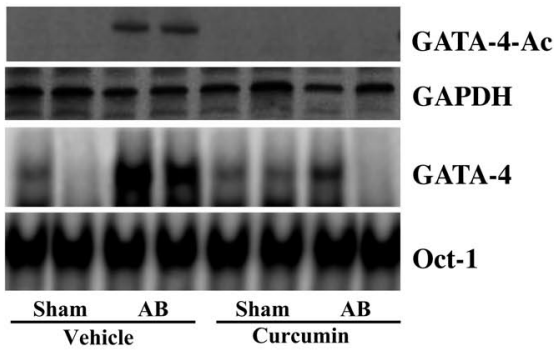

Figure 5

Pretreatment with curcumin blocks GATA4 activation. (A and B) Curcumin blocked the acetylation and DNA-binding activity of GATA4 induced by PE infusion (A) or AB (B). $n=4$. Oct-1 DNA-binding activity was used as a control. (C) Effect of p300 on the acetylation and DNA-binding activity of GATA4 induced by PE. Cells were infected with Ad-p300, Ad-DN-p300, or Ad-GFP for 24 hours and then treated with $100 \mu \mathrm{M}$ PE for 24 hours. Extracts were assayed for GATA4 acetylation and DNA-binding activity. (D) p300 partially reversed the inhibitory effect of curcumin on the acetylation and DNA-binding activity of GATA4 induced by PE. Cells were infected with Ad-p300 or Ad-GFP for 24 hours, treated with $25 \mu \mathrm{M}$ curcumin for 60 minutes, and then incubated with $100 \mu \mathrm{M}$ PE for 24 hours. The results were reproducible in 3 separate experiments.

increased in our Ad-p300-infected samples when compared with the Ad-GFP controls, which reached peak levels after 2 days and tapered off by 21 days (Figure $8 \mathrm{~A}$ ). Next, we studied the functional consequences of increased p300 expression. The Ad-GFP-infected mice demonstrated significant ventricular dilatation and decreased percent FS compared with the sham-operated mice after 2 weeks of AB (data not shown). Hypertrophied hearts treated with p300 gene transfer showed significant functional deterioration compared with those treated with GFP gene transfer after 2 weeks of $\mathrm{AB}$ (Figure 8B). Interestingly, curcumin treatment markedly attenuated the functional deterioration observed in Ad-GFP-infected mice but had no alleviating effect on Ad-p300-treated mice (Figure 8B). Furthermore, Ad-p300 infection also partially but obviously reversed the inhibitory effects of curcumin on the HW/BW ratio, cardiomyocyte cross-sectional area, and cardiac morphology after 2 weeks of $\mathrm{AB}$ (Figure 8, C and D). Northern blot analysis further revealed that $\mathrm{Ad}-\mathrm{p} 300$ infection significantly reversed the attenuated mRNA levels of ANF and BNP at 2 weeks after AB compared with those Ad-GFP-infected groups after treatment with curcumin (Figure 8E). These results suggest that p300 overexpression partially reverses the inhibitory effects of curcumin on cardiac hypertrophy. As inflammation and pathological fibrosis have been shown to be inhibited by curcumin, we determined whether overexpression of p300 might annul the inhibitory effects of curcumin on inflammation and fibrosis. Ad-p300 infection significantly reversed the inhibitory effect of curcumin on NF- $\kappa \mathrm{B}$ activation and NF- $\kappa \mathrm{B}-$ dependent TNF- $\alpha$ and IL- 6 expression (Supplemental Figure 5A and Figure 8F). Additionally, overexpression of p300 significantly $(10,11)$. However, to our knowledge there was no evidence to date regarding the effects of curcumin on cardiac hypertrophy. Despite significantly increased blood pressure in our 2 cardiac hypertrophy models, curcumin treatment did not affect blood pressure. This indicates that the primary target of curcumin action is cardiac protection, rather than lowering blood pressure. Of particular clinical relevance is the finding that curcumin can reverse preestablished cardiac hypertrophy and dysfunction induced by different animal models. The raw ingredient for curcumin is abundant and inexpensive, and the amount of curcumin used is within the physiologic range and well below the maximum tolerable pharmacological level (equivalent to $0.4 \mathrm{~g} / \mathrm{d}$ for humans).

The mechanism by which curcumin mediates its antihypertrophic effects remains unclear. There is increasing evidence for the involvement of chromatin remodeling, especially histone acetylation in pathological cardiac hypertrophy and heart failure (23-25). HATs are believed to acetylate histone proteins, relax chromatin, and expose prohypertrophic genes for activation by cardiogenic transcription factors. Several lines of evidence have shown that a critical HAT in the heart is p300, which plays a key role in the physiological growth and differentiation of cardiac myocytes during development (9). p300 knockout mice die between days 9 and 11.5 of gestation, exhibiting defects of cardiac muscle differentiation and trabeculation, indicating the importance of p300 for early cardiac morphogenesis and heart development (26). However, p300 is also involved in the pathological process of cardiac hypertrophy (8). The results of the present study indicate that inhibition of histone acetylation is a key mechanism for the antihypertrophic 
A

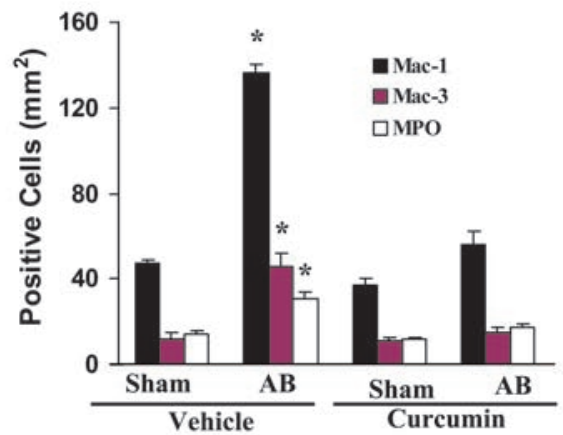

C

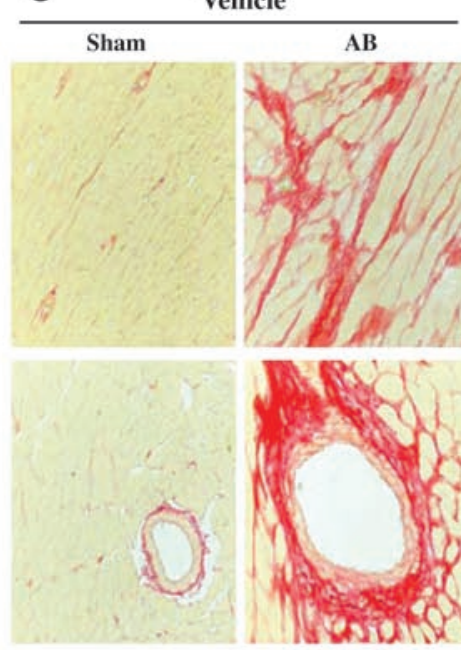

\section{E}

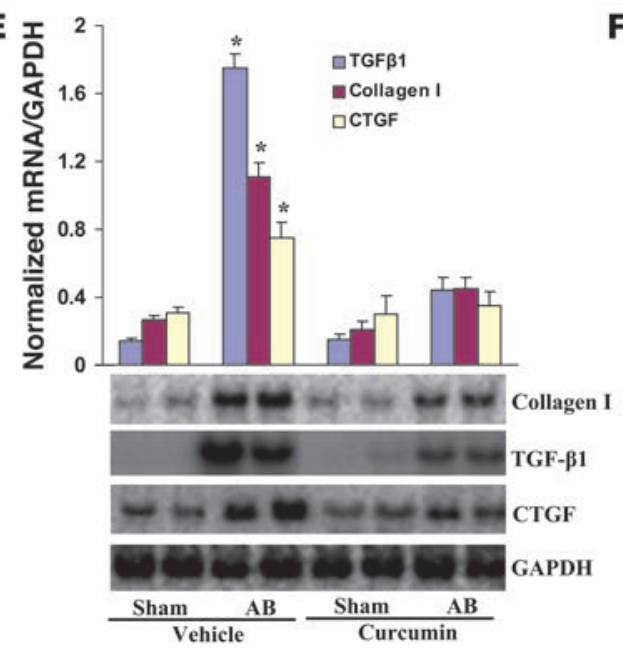

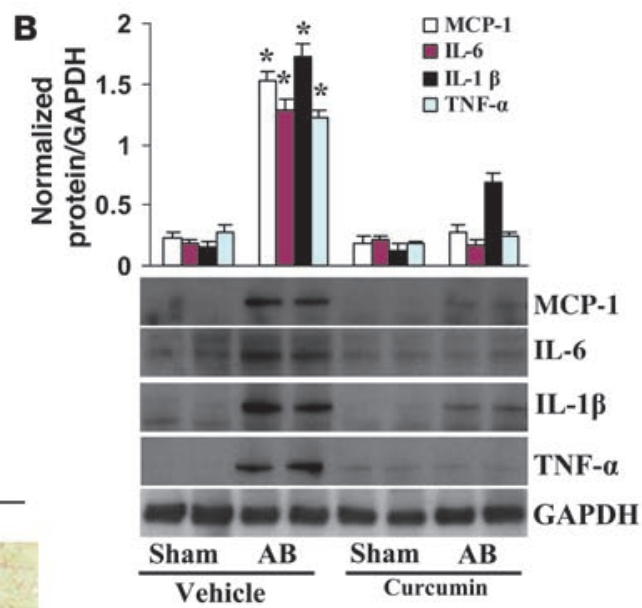

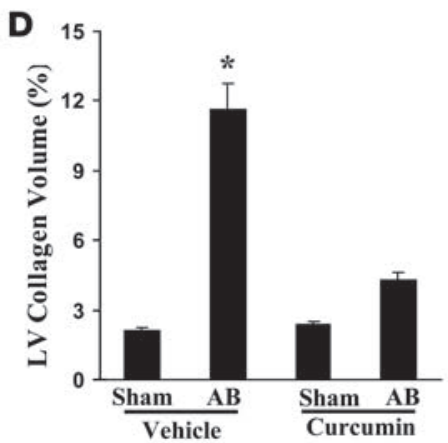

\section{Figure 6}

Pretreatment with curcumin inhibits inflammation and fibrosis induced by AB. (A) Quantitative analysis revealed the population of MPO-, Mac-1-, and Mac-3-positive cells in the hearts of the indicated mice $(n=6)$. (B) Western blot analysis of TNF- $\alpha$, IL-1 $\beta$, IL-6, and MCP-1 protein expression in the myocardia obtained from the indicated mice $(n=6)$. Each assay was performed in triplicate. (C) PSR staining on histological sections of the LV was performed on each group 8 weeks after AB. Scale bars: $10 \mu \mathrm{m}$. (D) Fibrotic areas from histological sections were quantified using an image-analyzing system $(n=6)$. (E and F) Northern blot and Western blot analyses of TGF- $\beta 1$, collagen I, and CTGF were performed to determine mRNA (E) and protein (F) expression levels in each group 8 weeks after AB $(n=3)$. GAPDH was used as the sample loading control. ${ }^{*} P<0.05$ versus vehicle-treated sham control.

activity of curcumin and that p300-HAT serves as its molecular target. We found that treatment with curcumin had a negligible effect on the normal heart, in contrast to its effects on the heart subjected to hemodynamic stress. In addition, we did not find any appreciable increase in cell death with curcumin treatment in the adult heart, suggesting that the role of p300 in the postnatal heart is different from its role during early cardiac morphogenesis. Consistent with our findings, Miyamoto et al. demonstrated 


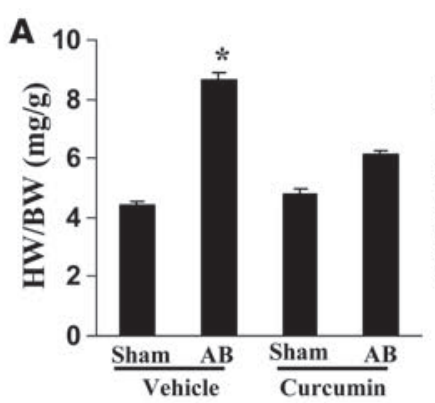

B

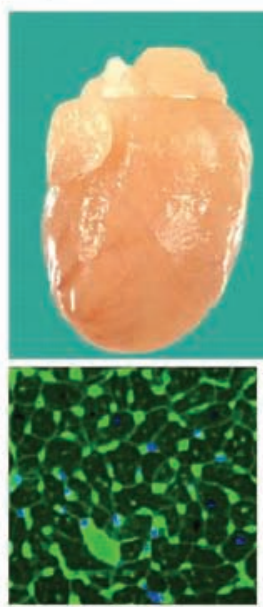

Sham
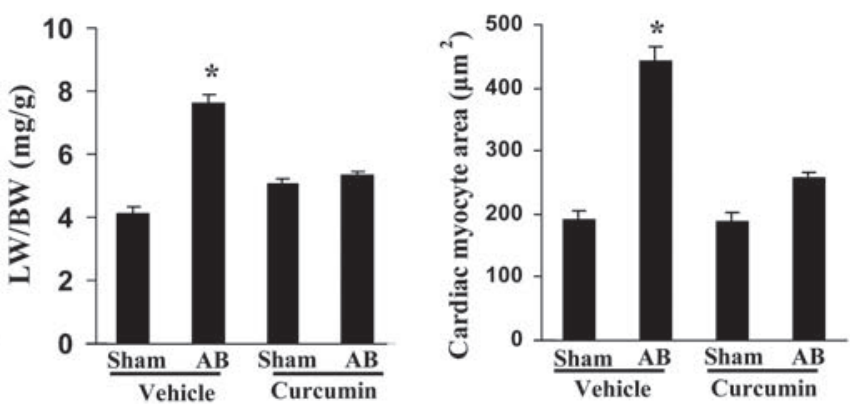

Curcumin 6 weeks

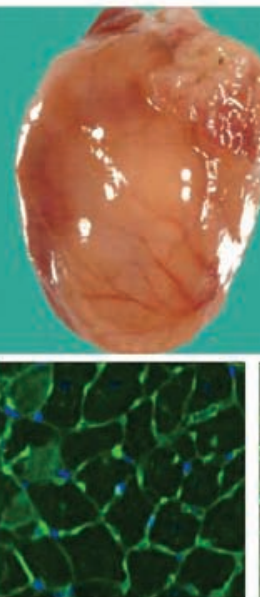

AB 8 weeks

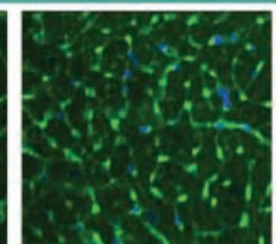

Sham
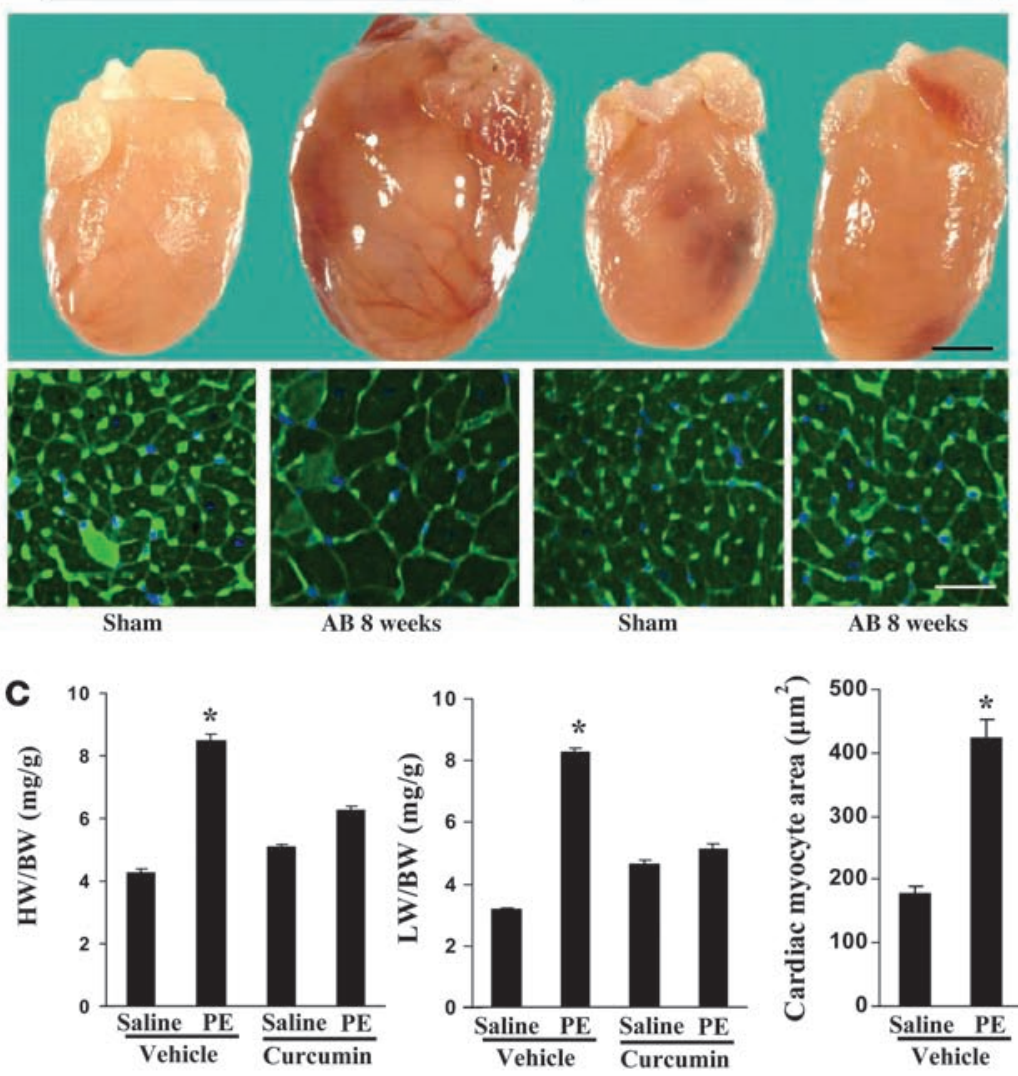

AB 8 weeks
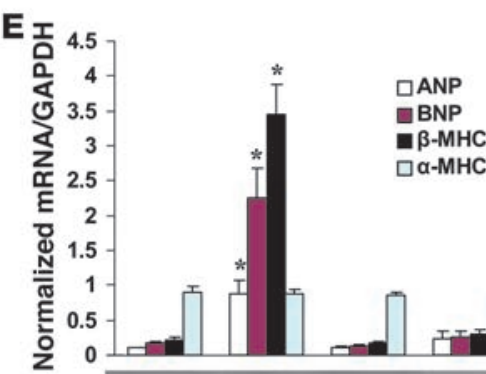

D

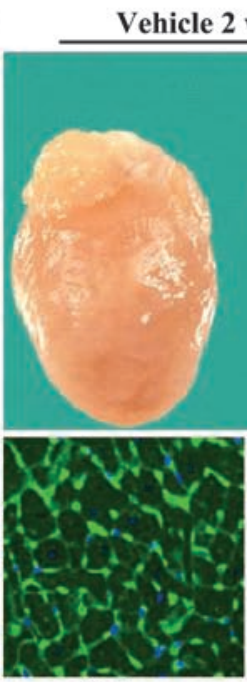

Saline

\section{Curcumin 2 weeks}

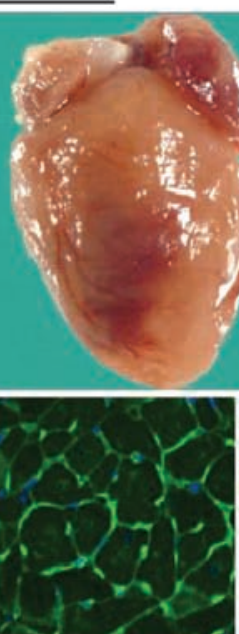

PE 3 weeks

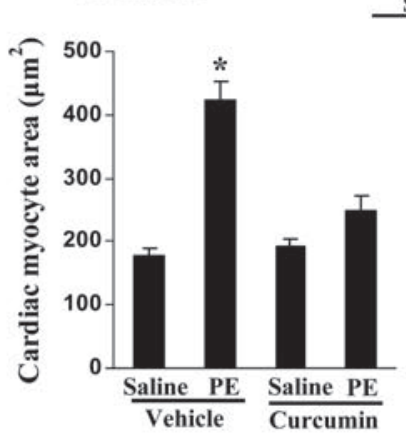




\section{Figure 7}

Curcumin ameliorates established cardiac hypertrophy. (A and $\mathbf{C}$ ) Statistical results of HW/BW ratio, LW/BW ratio, and myocyte crosssectional areas ( 200 cells per section) at 8 weeks after $A B$ surgery $(\mathbf{A}$; $n=5)$ or 3 weeks after PE infusion $(\mathbf{C} ; n=6)$. (A) Mice began treatment with curcumin at 2 weeks after $A B$ or sham surgery and then sacrificed 8 weeks later. (C) Mice began treatment with curcumin at 1 week after PE or saline infusion and were sacrificed at 3 weeks later. (B and D) Gross heart and WGA-FITC staining of mice 8 weeks after sham or AB surgery (B) or 3 weeks after saline or PE infusion (D) treated with or without curcumin. Scale bars: $20 \mathrm{~mm}$ (gross heart); 50 $\mu \mathrm{m}$ (WGA stain). ( $\mathrm{E}$ and F) Analysis of hypertrophic markers $(n=4)$. Total RNA was isolated from hearts of the indicated mice, and expression of transcripts for ANP, BNP, $\beta-\mathrm{MHC}$, and $\alpha-\mathrm{MHC}$ induced by $A B(E)$ or $P E$ infusion $(F)$ were determined by Northern blot analysis. ${ }^{\star} P<0.05$ versus respective vehicle control.

that cardiac-specific overexpression of wild-type $\mathrm{p} 300$ protein in the normal heart had no effect on cardiac morphology or function, in contrast to the resulting deteriorated function observed after myocardial infarction (9). We hypothesize that in the context of the postnatal myocardium, p300 may maintain basal function in the normal heart but promote cardiac growth under chronic loading stress or PE infusion. Importantly, in contrast to the complete depletion of p300 in knockout mice, elevated levels of p300 in cardiac hypertrophy were reduced (but not eliminated) with a therapeutic dose of curcumin in this study. The lack of physiological levels of p300-HAT activity during embryonic cardiac development or other organogenesis is not comparable with the suppression of elevated p300-HAT activity in adult animals with cardiac hypertrophy. Based on our present results, we believe curcumin to correct the disease-related increase in p300-HAT activity. Our data also indicate that curcumin inhibits p300-HAT activity but does not affect the activity of HDAC. Blocking p300-HAT activity completely blocks the inhibition by curcumin of histone acetylation, suggesting that the balance of HATs and HDACs is primarily modulated by curcumin through its effects on HAT activity. p300 acetylates GATA4 and increases its DNA-binding ability, leading to nuclear hyperacetylation of cardiac myocytes and cardiac hypertrophy (27-29). Consistent with recent reports (29), we observed GATA4 hyperacetylation and increased DNA-binding activity in response to hypertrophic stimuli. Curcumin treatment abolished GATA4 acetylation and attenuated DNA-binding activity in our hypertrophic models, and these effects were markedly reversed by p300 overexpression in vitro and in vivo. These findings suggest that curcumin blunts cardiac hypertrophy by inhibiting p300HAT-dependent GATA4 activation.

Inflammation plays an important role in the progression to cardiac hypertrophy (20), and perivascular infiltration by neutrophils and macrophages has been observed in the hearts of several animal species subjected to $\mathrm{AB}(30,31)$. After curcumin administration, we observed marked attenuation of leukocyte infiltration and cytokine production, suggesting potential cross-talk between leukocyte infiltration and inflammation in modulating the microenvironment for the development of cardiac hypertrophy. By blocking NF-кB signaling, curcumin may inhibit the early steps of inflammation and modulate the amplification of multiple cytokine signaling cascades (32-34). Acetylation is also an important modification step for the regulation of the nuclear function of $\mathrm{NF}-\kappa \mathrm{B}(22,35)$, and p300 appears to play a major role in the acetylation of p65 and subsequent regulation NF-אB activity (22). Our data suggest that suppression of $\mathrm{p} 300-\mathrm{HAT}$ activity results in impaired NF- $\mathrm{\kappa B}$ activity, while upregulation of $\mathrm{p} 300$-HAT activity promotes NF- $\kappa \mathrm{B}$ activation, indicating that $\mathrm{p} 300-\mathrm{HAT}$ plays an important role in the activation of NF- $\mathrm{KB}$ signaling. We have shown that curcumin can inhibit these signaling pathways in the heart.

Cardiac fibrosis is a classical feature of pathological hypertrophy and is characterized by the expansion of the extracellular matrix due to the accumulation of collagen (1). The present study demonstrated that curcumin blocks cardiac fibrosis in vivo and inhibits collagen synthesis in vitro. Our study is the first to our knowledge to report inhibition of TGF- $\beta 1$-induced collagen synthesis in cardiac fibroblasts by curcumin. Furthermore, our data suggest that curcumin abrogates Smad-2 phosphorylation and Smad-2/3/4 translocation in both cardiac fibroblast culture and hypertrophied hearts, important downstream components of TGF- $\beta 1$ signaling $(36,37)$. Recent studies indicate that TGF- $\beta 1-$ Smad signaling can be regulated by p300 $(37,38)$. Our findings demonstrated that blocking of p300-HAT activity led to complete inhibition, while forced expression of p300 led to upregulation, of collagen synthesis and Smad-2/3 activation. p300 gene transfer also markedly reversed the inhibitory effects of curcumin on fibrosis in vivo, indicating that the inhibitory effects of curcumin on fibrosis and collagen synthesis are mediated through blocking p300-HAT activity.

\section{Table 3}

Effects of curcumin on established cardiac hypertrophy induced by $A B$ model

Vehicle

$\begin{array}{lcccc}\text { Group }(\boldsymbol{n}) & \text { Basal (14) } & \mathbf{A B}, \mathbf{4} \mathbf{w k}(\mathbf{1 2}) & \mathbf{A B}, \mathbf{6} \text { wk (9) } & \mathbf{A B}, \mathbf{8} \text { wk (7) } \\ & & & & \\ \text { BW }(\mathrm{g}) & 25.3 \pm 1.4 & 27.5 \pm 1.7 & 28.7 \pm 1.3 & 26.8 \pm 2.1 \\ \text { SBP }(\mathrm{mmHg}) & 108.5 \pm 5.3 & 134.2 \pm 4.9^{\mathrm{A}} & 147.3 \pm 4.7^{\mathrm{A}} & 156.5 \pm 4.2^{\mathrm{A}} \\ \text { HR (bpm) } & 465 \pm 28 & 456 \pm 37 & 476 \pm 18 & 457 \pm 25 \\ \text { LVEDD (mm) } & 3.58 \pm 0.05 & 4.14 \pm 0.02^{\mathrm{A}} & 4.84 \pm 0.04^{\mathrm{A}} & 5.17 \pm 0.08^{\mathrm{A}} \\ \text { LVESD (mm) } & 1.67 \pm 0.05 & 2.58 \pm 0.04^{\mathrm{A}} & 3.21 \pm 0.05^{\mathrm{A}} & 3.69 \pm 0.06^{\mathrm{A}} \\ \text { IVSd (mm) } & 0.66 \pm 0.02 & 0.94 \pm 0.04 & 1.37 \pm 0.04^{\mathrm{A}} & 1.68 \pm 0.04^{\mathrm{A}} \\ \text { LVPWd (mm) } & 0.68 \pm 0.04 & 0.76 \pm 0.02 & 1.21 \pm 0.04^{\mathrm{A}} & 1.37 \pm 0.05^{\mathrm{A}} \\ \text { FS (\%) } & 55.6 \pm 2.3 & 41.7 \pm 1.3^{\mathrm{A}} & 31.0 \pm 2.2^{\mathrm{A}} & 17.8 \pm 3.3^{\mathrm{A}}\end{array}$

Curcumin, $75 \mathrm{mg} / \mathrm{kg} / \mathrm{d}$

$\begin{array}{cccc}\text { Basal (15) } & \begin{array}{c}\text { AB, } \mathbf{4} \text { wk }+ \\ \text { Cur, } \mathbf{w} \text { wk (13) }\end{array} & \begin{array}{c}\mathbf{A B}, \mathbf{6} \text { wk }+ \\ \text { Cur, } \mathbf{4} \text { wk (10) }\end{array} & \begin{array}{c}\text { AB, 8 wk }+ \\ \text { Cur, } \mathbf{6} \text { wk (7) }\end{array} \\ 25.3 \pm 1.0 & 25.8 \pm 2.4 & 27.6 \pm 1.8 & 27.3 \pm 1.5 \\ 109.4 \pm 2.5 & 136.7 \pm 3.2^{\mathrm{A}} & 147.3 \pm 3.3^{\mathrm{A}} & 150.9 \pm 3.8^{\mathrm{A}} \\ 447 \pm 35 & 467 \pm 26 & 475 \pm 11 & 442 \pm 36 \\ 3.81 \pm 0.01 & 3.86 \pm 0.04 & 3.92 \pm 0.03^{\mathrm{B}} & 4.24 \pm 0.03^{\mathrm{A}, \mathrm{B}} \\ 1.70 \pm 0.02 & 1.87 \pm 0.03^{\mathrm{B}} & 2.08 \pm 0.05^{\mathrm{B}} & 2.38 \pm 0.05^{\mathrm{A}, \mathrm{B}} \\ 0.67 \pm 0.05 & 0.83 \pm 0.04 & 0.91 \pm 0.04^{\mathrm{B}} & 1.03 \pm 0.02^{\mathrm{B}} \\ 0.70 \pm 0.02 & 0.72 \pm 0.03^{\mathrm{B}} & 0.78 \pm 0.03^{\mathrm{B}} & 0.94 \pm 0.04^{\mathrm{B}} \\ 54.6 \pm 1.7 & 48.2 \pm 1.9^{\mathrm{A}, \mathrm{B}} & 40.7 \pm 3.1^{\mathrm{A}, \mathrm{B}} & 38.5 \pm 2.5^{\mathrm{A}, \mathrm{B}}\end{array}$

All values are mean \pm SEM. Cur, curcumin; SBP, systolic blood pressure; IVSd, LV septum, diastolic; LVPWd, LV posterior wall, diastolic. AP < 0.05 versus basal. ${ }^{\mathrm{B}} P<0.05$ versus vehicle after $\mathrm{AB}$. 


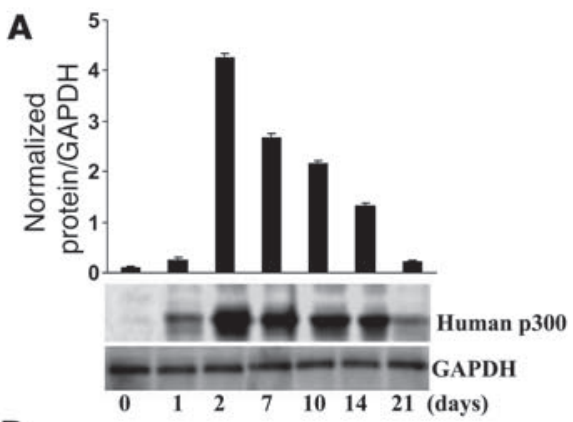

D

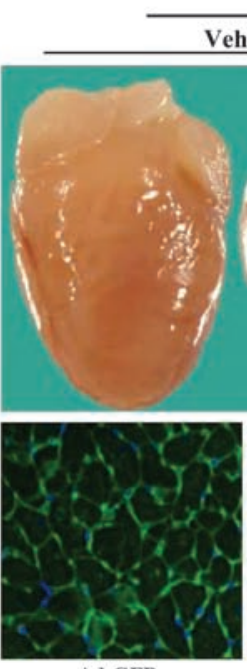

Ad-GFP

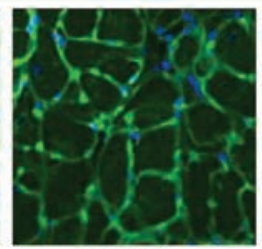

Ad-P300
AB for 2 weeks

\section{Curcumin}

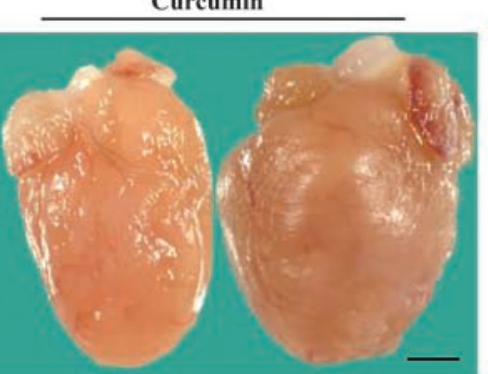

E

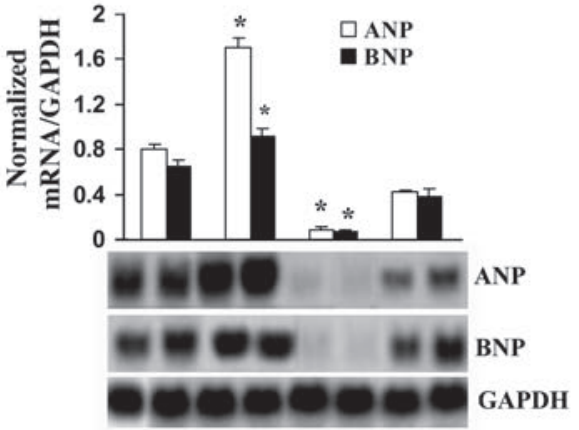

Ad-GFP

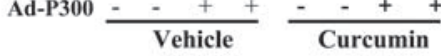

F

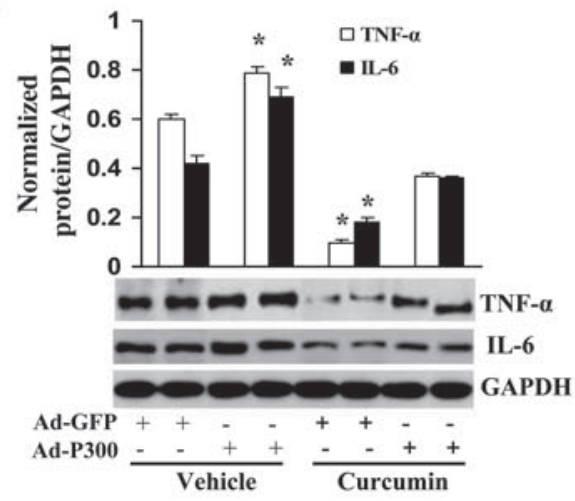

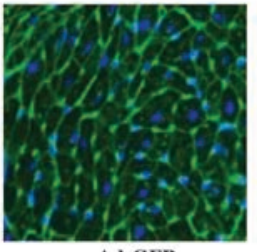

Ad-GFP

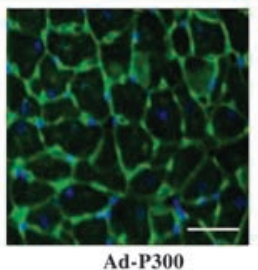

Ad-P300
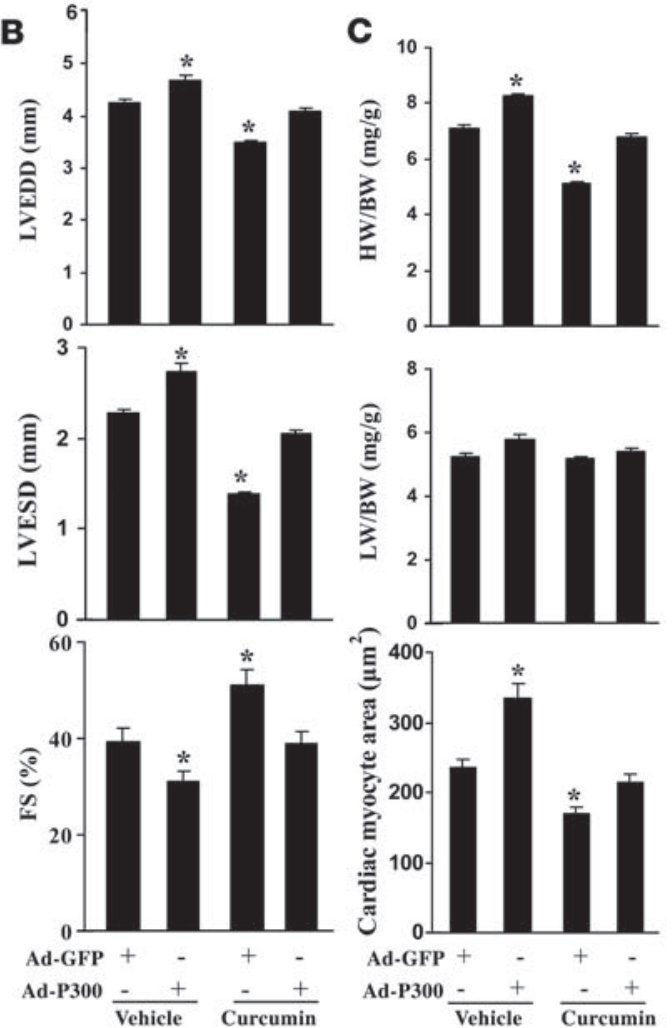

G

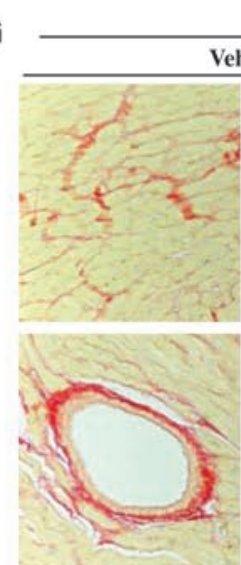

Ad-GFP
Vehicle

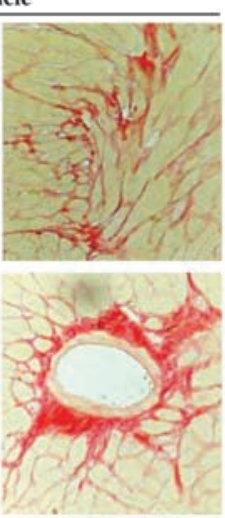

Ad-P300
AB for 2 weeks

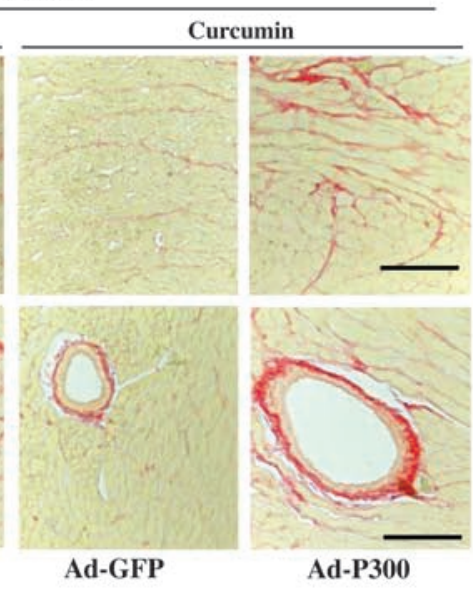

H

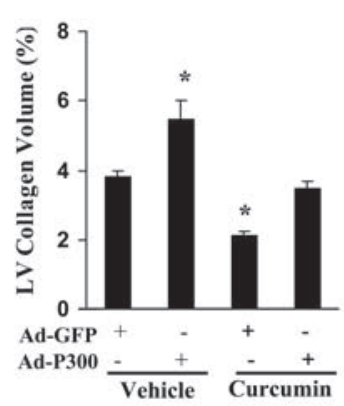




\section{Figure 8}

p300 partly reverses the inhibitory effects of curcumin on cardiac hypertrophy, inflammation, and fibrosis. (A) Human p300 protein expression $(n=4)$. Western blot showing adenoviral-mediated expression of human p300 protein for up to 21 days compared with Ad-GFP. Adenovirus $\left(2 \times 10^{9} \mathrm{pfu}\right)$ was injected into LV. Representative blots are shown. (B) Echocardiography results from 4 group mice at 2 weeks after AB surgery. (C) Statistical results of HW/BW ratio, LW/BW ratio, and myocyte cross-sectional areas ( $n=5 ; 200$ cells per section). (D) Gross heart and WGA staining of $A B$ mice at 2 weeks after surgery infected with Ad-p300 or Ad-GFP. Scale bar: $20 \mathrm{~mm}$ (gross heart); 50 $\mu \mathrm{m}$ (WGA stain). (E) Expression of ANP and BNP in hearts isolated from each group $(n=4)$. Representative blots are shown. (F) p300 partly reversed the inhibitory effects of curcumin on the protein expression of TNF- $\alpha$ and IL-6 $(n=4)$. Representative blots are shown. (G) PSR staining of the LV from paraffin-embedded histological sections from each group. Scale bars: $10 \mu \mathrm{m}$. (H) Quantification of fibrotic area measured by an image-analyzing system $(n=5)$. ${ }^{*} P<0.05$ versus Ad-GFP-infected vehicle control.

In conclusion, our present work demonstrates that curcumin inhibits cardiac hypertrophy in response to pathological stimuli both in vitro and in vivo. Curcumin prevented the development of cardiac hypertrophy by blocking p300-HAT-dependent hypertrophy, inflammation, and fibrosis. Our data confirm that p300-HAT is the main target of curcumin's inhibitory actions, although we cannot determine whether p300-HAT is its only target. This study is relevant to the understanding of the inhibitory effect of curcumin on cardiac hypertrophy and related molecular mechanisms. It also serves to elucidate the dominant signaling pathways leading to cardiac hypertrophy, inflammation, and fibrosis in response to hypertrophic stimuli. Curcumin is a natural polyphenolic compound that has already been used clinically and is approved by the FDA as a safe food additive. Future studies should examine the hypothesis that curcumin may be a safe and effective approach to preventing and treating cardiac hypertrophy and the transition to failure.

\section{Methods}

Materials. Anti-phospho-Smad2, anti-phospho-p65, anti-IKK $\alpha$, anti-IKK $\beta$,

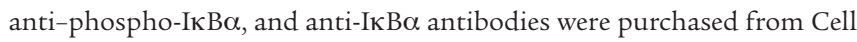
Signaling Technology. The antibodies used to recognize histones $\mathrm{H} 3$ and $\mathrm{H} 4$ at their $\mathrm{N}$-terminal lysine residues and GATA4 were purchased from Upstate Biotechnology. The anti-acetylated tubulin was obtained from Sigma-Aldrich. $\left[{ }^{3} \mathrm{H}\right]$ leucine, $\left[{ }^{3} \mathrm{H}\right]$ proline, $\left[{ }^{3} \mathrm{H}\right]$ acetate, and $\left[{ }^{3} \mathrm{H}\right]$ acetyl-CoA were purchased from Amersham. The BCA protein assay kit was purchased from Pierce, and the IKK activity kit was obtained from BD Biosciences. All other antibodies were purchased from Santa Cruz Biotechnology. TGF- $\beta 1$ was purchased from R\&D Systems. FCS was obtained from Hyclone. CTGF-luc and COL1A2-luc report constructs were provided by M. Trojanowska (Medical University of South Carolina, Charleston, South Carolina, USA), and PAI-1-luc report construct was provided by F.M. Stanley (New York University School of Medicine, New York, New York, USA). Ad-p300 and Ad-DN-p300 were provided by L. Hua (Oregon Health and Sciences University, Portland, Oregon, USA; ref. 39). Cell culture reagents, curcumin, and all other reagents were obtained from Sigma-Aldrich.

Cultured neonatal rat cardiac myocytes and fibroblasts. Primary cultures of cardiac myocytes were prepared as described previously (33). Cells from the hearts of 1- to 2-day-old Sprague-Dawley rats (Charles River Laboratories) were seeded at a density of $1 \times 10^{6}$ cells/well onto 6 -well culture plates coated with fibronectin (BD) in plating medium consisting of F10 medium supplemented with $10 \%$ FCS and penicillin/streptomycin. After 48 hours, the culture medium was replaced with F10 medium containing $0.1 \%$ FCS and $\mathrm{BrdU}(0.1 \mathrm{mM})$. After 12 hours of serum starvation, curcumin alone or curcumin followed by $100 \mu \mathrm{M}$ PE was added to the medium, and cultures were incubated for the indicated times. Viability was determined by cell number, frequency of contractions, cellular morphology, and trypan blue exclusion. Cultures of neonatal rat ventricular nonmyocytes, which have been shown to be predominantly fibroblasts, were prepared as described previously by Sadoshima and Izumo (40). All experiments were performed on cells from the first or second passages, which were placed in DMEM containing $0.1 \%$ FCS for 24 hours before the experiment. The purity of these cultures was greater than $95 \%$ cardiac fibroblasts, as determined by positive staining for vimentin and negative staining for smooth muscle actin and von Willebrand factor. For cell infection, cardiac myocytes or cardiac fibroblasts were cultured at $1 \times 10^{6}$ cells/well in 6-well plates and exposed to $2 \times 10^{8} \mathrm{pfu}$ of each virus in $1 \mathrm{ml}$ serum-free medium for 24 hours. The cells were then washed and incubated in serum-containing medium for 24 hours. All additional treatments to which cells were subjected are described in the figure legends.

$L D H$ release. Cardiac myocytes or cardiac fibroblasts in 6-well plates were preincubated with different concentrations of curcumin for 48 hours or $25 \mu \mathrm{M}$ curcumin for various time periods and then incubated with PE or TGF- $\beta 1$ for the indicated times. LDH activity was measured using an LDH assay kit according to the manufacturer's instructions (Cayman). The absorbance was determined at $492 \mathrm{~nm}$ in an ELISA reader. The percentage of $\mathrm{LDH}$ released from the cells was determined as the LDH activity in supernatant divided by the combined LDH activity in supernatant and cell lysate.

$\left[{ }^{3} \mathrm{H}\right]$ Leucine incorporation and surface area. $\left[{ }^{3} \mathrm{H}\right]$ Leucine incorporation was measured as described previously (33). Briefly, cardiac myocytes were pretreated with curcumin for 60 minutes and subsequently stimulated with $\mathrm{PE}(100 \mu \mathrm{M})$ and coincubated with $\left[{ }^{3} \mathrm{H}\right]$ leucine $(2 \mu \mathrm{Ci} / \mathrm{ml})$ for the indicated time. At the end of the experiment, the cells were washed with Hanks' solution, scraped off the well, and then treated with $10 \%$ trichloroacetic acid at $4{ }^{\circ} \mathrm{C}$ for 60 minutes. The precipitates were then dissolved in $\mathrm{NaOH}(1 \mathrm{~N})$ and subsequently counted with a scintillation counter. For surface areas, the cells were fixed with $3.7 \%$ formaldehyde in PBS, permeabilized in $0.1 \%$ Triton X-100 in PBS, and stained with $\alpha$-actinin (Sigma-Aldrich) at a dilution of 1:100 by standard immunocytochemical techniques.

Reporter assays. Cardiac myocytes or cardiac fibroblasts were seeded in triplicate in 6 -well plates. Cells were transfected with $0.5 \mu \mathrm{g}$ luciferase reporter constructs, and internal control plasmid DNA using $10 \mu \mathrm{l}$ of LipofectAMINE reagent (Invitrogen), according to the manufacturer's instructions. After 6 hours of exposure to the DNA-LipofectAMINE complex, cells were cultured in medium containing $10 \%$ serum for 18 hours and then incubated with serum-free medium for 12 hours. Cells were pretreated with curcumin for 60 minutes and then treated with PE for cardiac myocytes or TGF- $\beta 1$ for fibroblasts. Cells were harvested using passive lysis buffer (Promega) according to the manufacturer's protocol. The luciferase activity was normalized by control plasmid. All experiments were done in triplicate and repeated at least 3 times.

Collagen synthesis assay. Collagen synthesis was evaluated by measuring $\left[{ }^{3} \mathrm{H}\right]$ proline incorporation as described previously (41). In brief, cardiac fibroblasts were made quiescent by culturing in $0.1 \%$ FCS DMEM for 24 hours, pretreating with curcumin for 60 minutes and subsequently incubating with TGF- $\beta 1$ and $5 \mu \mathrm{Ci} / \mathrm{ml}\left[{ }^{3} \mathrm{H}\right]$ proline for the indicated time. Cells were washed with PBS twice, treated with ice-cold 5\% trichloroacetic acid for 1 hour, and washed with distilled water twice. Cells were then lysed with $1 \mathrm{~N} \mathrm{NaOH}$ solutions and counted in a liquid scintillation counter. The count representing the amount of newly synthesized collagen was normalized to the cell number. 
Quantitative real-time RT-PCR. Total RNA was extracted from frozen, pulverized mouse tissues using TRIzOL (Invitrogen) and synthesized cDNA using oligo(dT) primers with the Advantage RT-for-PCR kit (BD Biosciences). We quantified PCR amplifications using SYBR Green PCR Master Mix (Applied Biosystems) and normalized results against GAPDH gene expression.

Western blotting and Northern blot. Cardiac tissue and cultured cardiac myocytes or fibroblasts were lysed in RIPA lysis buffer. Nuclear protein extracts were isolated as described previously (21). Cell lysate $(50 \mu \mathrm{g})$ was used for SDS-PAGE, and proteins were then transferred to an Immobilon-P membrane (Millipore). Specific protein expression levels were normalized to either the GAPDH protein for total cell lysate and cytosolic protein or the lamin-B1 protein for nuclear protein signal on the same nitrocellulose membrane. For mRNA analysis, RNA was separated by $1 \%$ formaldehydeagarose gel electrophoresis and transferred to a NYTRAN SuPerCharge nylon membrane. Hypertrophy markers, fibrosis markers, and GAPDH probes were prepared by PCR as described previously (21) and labeled with $\alpha-\left[{ }^{32} \mathrm{P}\right] \mathrm{dCTP}$ using Prime-a-Gene labeling system. The radioactivity was detected by Phosphoimager using a scanner.

EMSA and IKK. Nuclear proteins were isolated as described previously (33). EMSA was performed according to the manufacturer's instructions (Gel Shift Assay System E3300; Promega). Synthetic, double-strand oligonucleotides containing NF-KB and GATA4 binding domains were labeled with $\left[\gamma^{-32} \mathrm{P}\right]$ ATP using $\mathrm{T}_{4}$ polynucleotide kinase and separated from unincorporated $\left[\gamma^{-32} \mathrm{P}\right]$ ATP by gel filtration using a Nick column (Pharmacia). IKK complexes were isolated from mouse heart lysates and cultured cardiac myocytes using anti-IKK $\gamma$ antibodies (BD Biosciences - Pharmingen) coupled to protein A sepharose, and analyzed using in vitro kinase assays, performed at $30^{\circ} \mathrm{C}$ with $\left[\gamma^{-32} \mathrm{P}\right] \mathrm{ATP}$ and $3 \mu \mathrm{g}$ GST-IKB $\alpha$.

Histone acetylation assay and histone deacetylation activity. For the global acetylation of histones, cells were plated at a density of $1 \times 10^{6}$ cells $/ \mathrm{ml}$ and exposed to the indicated amounts of curcumin in the presence of 10 $\mu \mathrm{Ci} / \mathrm{ml}\left[{ }^{3} \mathrm{H}\right]$ acetate $(5.0 \mathrm{Ci} / \mathrm{mmol})$ for the indicated times. Before collecting the cells, TSA was used (or not) to stimulate the cells for 6 hours. Histones were then isolated, and ${ }^{3} \mathrm{H}$-labeled histones were determined by liquid scintillation counting. To analyze the acetylation of histone $\mathrm{H} 3$, histone $\mathrm{H} 4$, or tubulin, histones from cardiac tissue and cardiac myocytes were prepared as described previously (42). The prepared histones were suspended in $4 \mathrm{M}$ urea and stored at $-20^{\circ} \mathrm{C}$ until used. Equal amounts of histones $(10 \mu \mathrm{g})$ were subjected to SDS-PAGE on $15 \%$ polyacrylamide gels and were electrophoretically transferred to a nitrocellulose membrane. Nitrocellulose blots were blocked with $5 \%$ milk in TTBS (Tris-buffered saline plus $0.05 \%$ Tween 20 , pH 7.5) and incubated overnight at $4^{\circ} \mathrm{C}$ with an antibody against acetyl-histone $\mathrm{H} 3$, histone $\mathrm{H} 4$, or tubulin in TTBS containing $5 \%$ milk. After incubation with horseradish peroxidase-conjugated secondary antibody, immunoreactivity was visualized by means of enhanced chemiluminescence. GATA4 acetylation was detected as described previously (43). HDAC activity was measured by colorimetric HDAC assay kit (ab1438; Abcam) as described previously (25). The absorbance of samples is expressed as arbitrary units equivalent to the absorbance obtained with specific concentrations of deacetylated standard.

Immunoprecipitation HAT assay. HAT assays were performed using antip300 antibody following the manufacturer's protocol (Upstate) as described previously (42). Briefly, cardiac myocytes were collected, pelleted, and resuspended in RIPA lysis buffer after various treatments. Protein concentrations were determined using BCA Protein Assay. For immunoprecipitation, 500 $\mu \mathrm{g}$ lysate proteins were precipitated with anti-p300 antibody (Upstate) and incubated at $4^{\circ} \mathrm{C}$ overnight. Protein $\mathrm{G}$ sepharose beads $(30 \mu \mathrm{l})$ were added and incubated at $4^{\circ} \mathrm{C}$ for 1 hour. HAT assay cocktail $(50 \mu \mathrm{l})$ containing $10 \mu \mathrm{l}$ biotinylated histone $\mathrm{H} 4$ peptide was added to the immunoprecipitated p300. The mixture was then incubated at $30^{\circ} \mathrm{C}$ for 1 hour in a shaking incubator. $\left[{ }^{3} \mathrm{H}\right]$ acetyl incorporation into the substrates was determined by counting in a liquid scintillation counter.

Animal models. All protocols were approved by the Animal Care and Use Committee of University Health Network (Toronto, Ontario, Canada). All surgeries and subsequent analyses were performed in a blinded fashion for all groups. The adult male C57BL/6 mice (8-10 weeks old) used in the current study were purchased from the Jackson Laboratory and acclimatized for 1 week prior to experimental use. $\mathrm{AB}$ was performed as described previously (44). Doppler analysis was performed to ensure that physiologic constriction of the aorta was induced. To further detect the effects of curcumin on cardiac hypertrophy, PE infusion models were performed. PE $(65 \mathrm{mg} / \mathrm{kg} / \mathrm{d}$ dissolved in $0.9 \% \mathrm{NaCl})$ was subcutaneously infused for 3 weeks using an osmotic minipump (Alzet model 2004; Alza Corp.) implanted in each mouse. Saline-infused animals served as infusion controls and were subjected to the same procedures as the experimental animals with the exception of PE infusion. Cardiac adenoviral delivery was performed according to the method of Lebeche et al. (45). With preliminary experiments demonstrating maximal p300 expression at 2 days after cardiac gene transfer, all subsequent experiments were carried out with the transfer of Ad-GFP or Ad-p300 2 days prior to AB. Curcumin suspension was prepared using $0.5 \%$ carboxy methylcellulose solution for animal experiments. Suspensions were freshly prepared and administered at a constant volume of $1 \mathrm{ml} / 100 \mathrm{~g} \mathrm{BW}$ by oral gavage 3 times a day. The control group of these animal experiments was given the same volume of liquid but composed solely of the vehicle solution ( $0.5 \%$ carboxy methylcellulose). The internal diameter and wall thickness of LV were assessed by echocardiography at the indicated times after surgery or infusion. Hearts and lungs of the sacrificed mice were dissected and weighed to compare $\mathrm{HW} / \mathrm{BW}(\mathrm{mg} / \mathrm{g})$ and $\mathrm{LW} / \mathrm{BW}(\mathrm{mg} / \mathrm{g})$ ratios in curcumin- and vehicle-treated mice.

Blood pressure and echocardiography. A microtip catheter transducer (SPR-839; Millar Instruments) was inserted into the right carotid artery and advanced into the LV under pressure control. After stabilization for 15 minutes, the pressure signals and heart rate were recorded continuously with an ARIA pressure-volume conductance system coupled with a Powerlab/4SP A/D converter, stored, and displayed on a personal computer as described previously (44). Echocardiography was performed by SONOS 5500 ultrasound (Philips Electronics) with a $15-\mathrm{MHz}$ linear array ultrasound transducer. The LV was assessed in both parasternal long-axis and short-axis views at a frame rate of $120 \mathrm{~Hz}$. End-systole or end-diastole was defined as the phase in which the smallest or largest LV area, respectively, was obtained. LVEDD and LVESD were measured from the LV M-mode tracing with a sweep speed of $50 \mathrm{~mm} / \mathrm{s}$ at the midpapillary muscle level.

Histological analysis. Hearts were excised, washed with saline solution, and placed in $10 \%$ formalin. Hearts were cut transversely close to the apex to visualize the LV and RV. Several sections of heart (4-5 $\mu \mathrm{m}$ thick) were prepared and stained with H\&E for histopathology or PSR for collagen deposition and then visualized by light microscopy. For myocyte cross-sectional area, sections were stained for membranes with FITC-conjugated WGA (Invitrogen) and for nuclei with DAPI. A single myocyte was measured with an image quantitative digital analysis system (NIH Image version 1.6). The outline of 100-200 myocytes was traced in each section. To identify infiltrating mononuclear cell populations, sections were incubated with anti-Mac-1 and anti-Mac-3 (BD) antibody for macrophages and anti-MPO (Abcam) antibody for neutrophils. Quantitative assessments for MPO-, Mac-1-, and Mac-3-positive cells were performed, and the number of positive cells in 5 randomly selected fields of view was calculated for each animal.

Statistics. Data are expressed as means \pm SEM. Differences among groups were tested by 1-way ANOVA. Comparisons between 2 groups were performed by unpaired Student's $t$ test. A $P$ value less than 0.05 was considered significant. 


\section{Acknowledgments}

We thank Maria Trojanowska for CTGF-luc and COL1A2-luc report constructs; Frederick M. Stanley for PAI-1-luc report construct; and Lu Hua for Ad-p300 and Ad-DN-p300. P. Liu is the Heart and Stroke/Polo Chair Professor of Medicine and Physiology of the University of Toronto and Scientific Director, Institute of Circulatory and Respiratory Health, of CIHR. L. Kirshenbaum is a Canada Research Chair in Molecular Cardiology at the University of Manitoba. The present study was supported in part by grants from the Heart and Stroke Foundation of Ontario, the CIHR, and the Team Research Program (CHENET) and Group Program (CHF-CORE) from CIHR.

Received for publication May 31, 2007, and accepted in revised form December 19, 2007.

Address correspondence to: Peter P. Liu, Institute of Circulatory and Respiratory Health, CIHR, NCSB 11-1266, Toronto General Hospital, 200 Elizabeth Street, Toronto, Ontario M5G2C4, Canada. Phone: (416) 340-3035; Fax: (416) 340-4753; E-mail: peter.liu@utoronto.ca.
1. Berk, B.C., Fujiwara, K., and Lehoux, S. 2007. ECM remodeling in hypertensive heart disease. J. Clin. Invest. 117:568-575.

2. Heineke, J., and Molkentin, J.D. 2006. Regulation of cardiac hypertrophy by intracellular signaling pathways. Nat. Rev. Mol. Cell Biol. 7:589-600.

3. Chen, F., et al. 2002. Hop is an unusual homeobox gene that modulates cardiac development. Cell. 110:713-723.

4. Backs, J., and Olson, E.N. 2006. Control of cardiac growth by histone acetylation/deacetylation. Circ. Res. 98:15-24.

5. Clayton, A.L., Hazzalin, C.A., and Mahadevan, L.C. 2006. Enhanced histone acetylation and transcription: a dynamic perspective. Mol Cell. 23:289-296.

6. Yanazume, T., et al. 2003. Cardiac p300 is involved in myocyte growth with decompensated heart failure. Mol. Cell. Biol. 23:3593-3606.

7. Chan, H.M., and La Thangue, N.B. 2001. p300/CBP proteins: HATs for transcriptional bridges and scaffolds. J. Cell. Sci. 114:2363-2373.

8. Gusterson, R.J., et al. 2003. The transcriptional coactivator CREB-binding protein (CBP) and p300 play a critical role in cardiac hypertrophy that is dependent on their histone acetyltransferase activity. J. Biol. Chem. 278:6838-6847.

9. Miyamoto, S., et al. 2006. Histone acetyltransferase activity of p300 is required for the promotion of left ventricular remodeling after myocardial infarction in adult mice in vivo. Circulation. 113:679-690.

10. Maheshwari, R.K., Singh, A.K., Gaddipati, J., and Srimal, R.C. 2006. Multiple biological activities of curcumin: a short review. Life Sci. 78:2081-2087.

11. Miquel, J., et al. 2002. The curcuma antioxidants: pharmacological effects and prospects for future clinical use. Arch. Gerontol. Geriatr. 34:37-46.

12. Balasubramanyam, K., et al. 2004. Curcumin, a novel p300/CREB-binding protein-specific inhibitor of acetyltransferase, represses the acetylation of histone/nonhistone proteins and histone acetyltransferase-dependent chromatin transcription. J. Biol. Chem. 279:51163-51171.

13. Mai, A., et al. 2006. Small-molecule inhibitors of histone acetyltransferase activity: identification and biological properties. J. Med. Chem. 49:6897-6907.

14. Dorn, G.W., 2nd, and Force, T. 2005. Protein kinase cascades in the regulation of cardiac hypertrophy. J. Clin. Invest. 115:527-537.

15. Perkins, S., et al. 2002. Chemopreventive efficacy and pharmacokinetics of curcumin in the $\mathrm{min} /+$ mouse, a model of familial adenomatous polyposis. Cancer Epidemiol. Biomarkers Prev. 11:535-540.

16. Sharma, R.A., et al. 2004. Phase I clinical trial of oral curcumin: biomarkers of systemic activity and compliance. Clin. Cancer Res. 10:6847-6854.

17. Dai, Y.S., and Markham, B.E. 2001. P300 Functions as a coactivator of transcription factor GATA-4. J. Biol. Chem. 276:37178-37185.

18. Zeisberg, E.M., et al. 2005. Morphogenesis of the right ventricle requires myocardial expression of Gata4. J. Clin. Invest. 115:1522-1531.

19. Li, H.L., et al. 2007. Overexpression of myofibrillogenesis regulator-1 aggravates cardiac hypertrophy induced by angiotensin II in mice. Hypertension. 49:1399-1408.

20. Nian, M., Lee, P., Khaper, N., and Liu, P. 2004. Inflammatory cytokines and postmyocardial infarction remodeling. Circ. Res. 94:1543-1553.

21. Li, H.L., et al. 2007. Targeted cardiac overexpression of A20 improves left ventricular performance and reduces compensatory hypertrophy after myocardial infarction. Circulation. 115:1885-1894.

22. Hoberg, J.E., Popko, A.E., Ramsey, C.S., and Mayo, M.W. 2006. IkappaB kinase alpha-mediated derepression of SMRT potentiates acetylation of RelA/ p65 by p300. Mol. Cell. Biol. 26:457-471.

23. Trivedi, C.M., et al. 2007. Hdac2 regulates the cardiac hypertrophic response by modulating Gsk3 beta activity. Nat. Med. 13:324-331.

24. Kook, H., and Epstein, J.A. 2003. Hopping to the beat. Hop regulation of cardiac gene expression. Trends Cardiovasc. Med. 13:261-264.

25. Kook, H., et al. 2003. Cardiac hypertrophy and histone deacetylase-dependent transcriptional repression mediated by the atypical homeodomain protein Hop. J. Clin. Invest. 112:863-871.

26. Yao, T.P., et al. 1998. Gene dosage-dependent embryonic development and proliferation defects in mice lacking the transcriptional integrator p300. Cell. 93:361-372.

27. Dai, Y.S., Cserjesi, P., Markham, B.E., and Molkentin, J.D. 2002. The transcription factors GATA4 and dHAND physically interact to synergistically activate cardiac gene expression through a 3300 -dependent mechanism. J. Biol. Chem. 277:24390-24398.

28. Epstein, J.A., and Parmacek, M.S. 2005. Recent advances in cardiac development with therapeutic implications for adult cardiovascular disease. Circulation. 112:592-597.

29. Bisping, E., et al. 2006. Gata4 is required for maintenance of postnatal cardiac function and protection from pressure overload-induced heart failure. Proc. Natl. Acad. Sci. U. S. A. 103:14471-14476.

30. Kuster, G.M., et al. 2005. Mineralocorticoid receptor inhibition ameliorates the transition to myocardial failure and decreases oxidative stress and inflammation in mice with chronic pressure overload. Circulation. 111:420-427.
31. Fedak, P.W., et al. 2004. TIMP-3 deficiency leads to dilated cardiomyopathy. Circulation. 110:2401-2409. 32. Li, H.L., et al. 2007. Long-term activation of adenosine monophosphate-activated protein kinase attenuates pressure-overload-induced cardiac hypertrophy. J. Cell. Biochem. 100:1086-1099.

33. Li, H.L., et al. 2006. Epigallocathechin-3 gallate inhibits cardiac hypertrophy through blocking reactive oxidative species-dependent and -independent signal pathways. Free Radic. Biol. Med. 40:1756-1775.

34. Li, H.L., et al. 2005. Isorhapontigenin, a new resveratrol analog, attenuates cardiac hypertrophy via blocking signaling transduction pathways. Free Radic. Biol. Med. 38:243-257.

35. Tak, P.P., and Firestein, G.S. 2001. NF-kappaB: a key role in inflammatory diseases. J. Clin. Invest. 107:7-11.

36. Bhattacharyya, S., et al. 2005. Fibroblast expression of the coactivator $\mathrm{p} 300$ governs the intensity of profibrotic response to transforming growth factor beta. Arthritis Rheum. 52:1248-1258.

37. Ghosh, A.K., et al. 2004. The tumor suppressor p53 abrogates Smad-dependent collagen gene induction in mesenchymal cells. J. Biol. Chem. 279:47455-47463.

38. Holmes, A., et al. 2001. CTGF and SMADs, maintenance of scleroderma phenotype is independent of SMAD signaling. J. Biol. Chem. 276:10594-10601.

39. MacPartlin, M., et al. 2005. p300 regulates p63 transcriptional activity. J. Biol. Chem. 280:30604-30610.

40. Sadoshima, J., and Izumo, S. 1993. Molecular characterization of angiotensin II-induced hypertrophy of cardiac myocytes and hyperplasia of cardiac fibroblasts. Critical role of the AT1 receptor subtype. Circ. Res. 73:413-423.

41. Dubey, R.K., Gillespie, D.G., and Jackson, E.K. 1998. Adenosine inhibits collagen and protein synthesis in cardiac fibroblasts: role of A2B receptors. Hypertension. 31:943-948.

42. Gong, J., et al. 2006. Activation of p300 histone acetyltransferase activity and acetylation of the androgen receptor by bombesin in prostate cancer cells. Oncogene. 25:2011-2021.

43. Kawamura, T., et al. 2005. Acetylation of GATA-4 is involved in the differentiation of embryonic stem cells into cardiac myocytes. J. Biol. Chem. 280:19682-19688.

44. Sun, M., et al. 2007. Tumor necrosis factor-alpha mediates cardiac remodeling and ventricular dysfunction after pressure overload state. Circulation. 115:1398-1407.

45. Lebeche, D., et al. 2004. In vivo cardiac gene transfer of $\mathrm{Kv} 4.3$ abrogates the hypertrophic response in rats after aortic stenosis. Circulation. 110:3435-3443. 